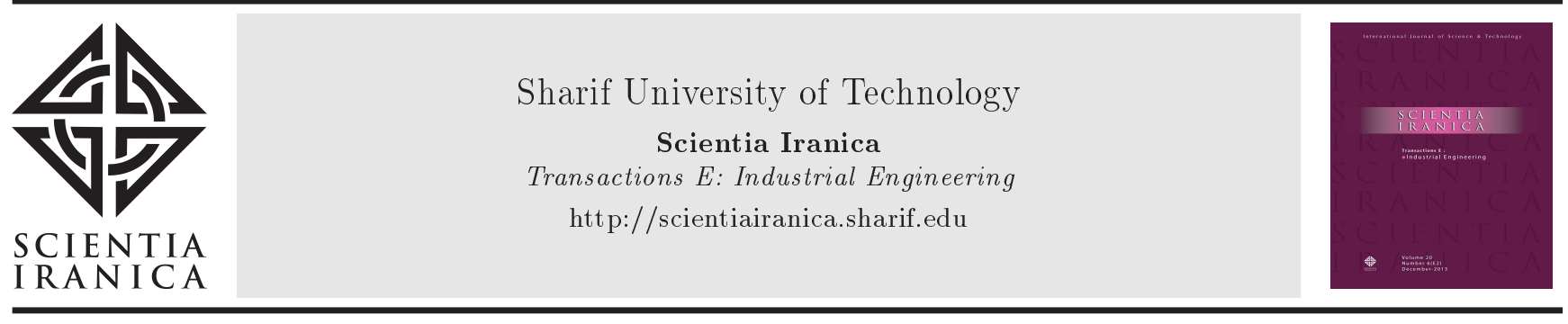

\title{
New Shewhart-EWMA and Shewhart-CUSUM control charts for monitoring process mean
}

\author{
M. Awais and A. Haq* \\ Department of Statistics, Quaid-i-Azam University, Islamabad, Pakistan.
}

Received 7 August 2017; received in revised form 19 February 2018; accepted 2 July 2018

\section{KEYWORDS}

Average run length; CUSUM;

Control chart;

EWMA;

Perfect and imperfect rankings;

Statistical process

control.

\begin{abstract}
This study proposes new Shewhart-EWMA (SEWMA) and Shewhart-CUSUM (SCUSUM) control charts using Varied L Ranked Set Sampling (VLRSS) for monitoring the process mean, namely the SEWMA-VLRSS and SCUSUM-VLRSS charts. The run length characteristics of the proposed charts are computed using extensive Monte Carlo simulations. The proposed charts are compared with their existing counterparts in terms of the average and standard deviations of run lengths. It is found that, with perfect and imperfect rankings, the SEWMA-VLRSS and SCUSUM-VLRSS charts are more sensitive than their analogous charts based on simple random sampling, Ranked Set Sampling (RSS), and median RSS schemes. A real dataset is also used to explain the implementation of the proposed control charts.
\end{abstract}

(C) 2019 Sharif University of Technology. All rights reserved.

\section{Introduction}

The Statistical Process Control (SPC) is a collection of tools that help distinguish between two types of variation, namely the natural- and special-cause variations. A process is said to be in statistical control when only natural-caused variations are present, while the process with the special-cause variations is said to be out of control. There are seven major tools in the SPC, including the histogram, check sheet, Pareto chart, cause-and-effect diagram, defect concentration diagram, scatter diagram, and control charts. Statistical quality control charts are very effective SPC tools that are frequently used to monitor special-cause variations in a production/manufacturing process.

The control charts are divided into two categories, memory-less and memory-type control charts. The

\footnotetext{
*. Corresponding author.

E-mail address: aaabdulhaq@yahoo.com (A.Haq)
}

Shewhart-type charts fall in the memory-less category because they completely rely on the present information. A major limitation of the Shewhart control chart is that it is less sensitive against small and moderate shifts in the process parameter(s). On the other hand, the Exponentially Weighted Moving Average (EWMA) and the CUmulative SUM (CUSUM) control charts fall in the memory-type category. The reason is that both of these control charts take into account past and current information to maintain their plotting statistics. This feature of the memory-type control charts helps them swiftly react against small to moderate shifts in the process parameter(s).

The CUSUM chart was first developed by Page [1]. Lucas and Crosier [2] associated a Fast Initial Response (FIR) feature with the CUSUM chart to further enhance its sensitivity by giving head-starts to the plotting CUSUMs at the beginning of a process. Lucas [3] used both the Shewhart and CUSUM charts simultaneously for monitoring small and large shifts in a process, named the Shewhart-CUSUM (SCUSUM) chart. For monitoring changes in the process mean, 
Roberts [4] was the first to introduce the EWMA chart. Lucas and Saccucci [5] attached an FIR feature with the EWMA chart to increase its sensitivity to the start-up/initial problems. Moreover, they coupled the Shewhart chart with the EWMA chart, named the Shewhart-EWMA (SEWMA) chart, for detecting small and large shifts simultaneously. There are many new advancements and improvements in the control charting structures of the EWMA and CUSUM charts. For some related works on these control charts, we refer to Knoth [6], Lucas and Crosier [2], Chiu [7], Abbas et al. [8], Haq [9], Haq et al. [10], and the references cited therein.

Ranked-Set Sampling (RSS) was first introduced by McIntyre [11] for estimating mean pasture and forage yields. RSS is a cost-effective alternative to Simple Random Sampling (SRS) in situations where the units to be sampled could be ranked with respect to each other prior to the formal measurements. Ranking may be done visually based on personal judgment or using the ranks of an auxiliary variable, provided that it is highly correlated with the study variable. For example, if the quantification of the underlying quality characteristic(s) is laborious, costly and timeconsuming, it may involve breaking the product-which is expensive and might be hard to construct, etc.-but using some experts' knowledge, it might be possible to rank the quality characteristic according to its quality level or using any less expensive method. There also exist the situations where the error is inevitable while ranking the units, particularly when ranking the units in large set sizes. Dell and Clutter [12] showed that, despite the presence of ranking errors, the mean estimator with RSS is not only unbiased but also more precise than the mean estimator with SRS. A simple imperfect ranking model was designed by Stokes [13], whereby the study variable could be ranked using the ranks of an auxiliary variable. For precisely estimating the mean of a symmetric population, Extreme RSS (ERSS) and Median RSS (MRSS) schemes were suggested by Samawi et al. [14] and Muttlak [15], respectively. The mean estimator with MRSS is more precise than those with the SRS and RSS, when sampling from a symmetric population. Muttlak [16] suggested using the Quartile RSS (QRSS) as a better alternative to SRS, RSS, and MRSS schemes for estimating the population mean when sampling from an asymmetric population. Utilizing the idea of L moments, Al-Nasser [17] proposed a generalized sampling scheme, named L RSS (LRSS), for estimating the population mean. The LRSS scheme encompasses existing RSS schemes, such as the RSS, QRSS, ERSS, and MRSS. Haq et al. [18] further extended the work of Al-Nasser [17] and generalized the LRSS scheme for efficiently estimating the population mean, named the Varied LRSS (VLRSS) scheme. For a symmetric population, the VLRSS scheme-with both perfect and imperfect rankings-not only is a cost-effective alternative to the exiting ranking schemes, but it also encompasses them, i.e., the mean estimator with the VLRSS scheme is better than the mean estimator based on SRS, RSS, ERSS, MRSS, and QRSS. For more details, we refer to Haq et al. [18].

As the mean estimators with the RSS schemes are more precise than the mean estimator based on SRS scheme, this fact has led many researchers to construct more sensitive quality control charts. Salazar and Sinha [19] were the first to propose a Shewhart chart using RSS for monitoring the process mean. Their work, later on, extended by Muttlak and AlSabah [20], who suggested several Shewhart-type mean charts using the RSS, ERSS, and MRSS schemes under both perfect and imperfect rankings. Abujiya and Muttlak [21] and Al-Omari and Haq [22] used the double RSS schemes to construct the Shewhart charts for monitoring the process mean. Al-Sabah [23] suggested new CUSUM mean charts using RSS and MRSS. He showed that the CUSUM charts with RSS and MRSS schemes were more sensitive than that using SRS. Recently, Abujiya et al. [24,25] proposed SEWMA and SCUSUM mean charts using RSS and MRSS schemes. In another work, Awais and Haq [26,27] have suggested improved EWMA and CUSUM charts for monitoring the process mean, respectively. For more related works on the RSS-based control charts, we refer to Haq [28], Mehmood et al. [29,30], Haq et al. [31-36], Abbasi and Riaz [37], Abid et al. [38,39], Munir and $\mathrm{Haq}$ [40], and the references cited therein.

Since the VLRSS mean estimator is more precise than the mean estimators based on SRS, RSS, and MRSS schemes when sampling from a symmetric population, we believe that the control charts with the VLRSS would be more sensitive than those based on SRS, RSS, and MRSS schemes. This paper proposes new SEWMA and SCUSUM mean charts using VLRSS, named SEWMA-VLRSS and SCUSUMVLRSS charts, respectively. Monte Carlo simulations are used to compute the run length characteristics of the proposed control charts, including the Average Run Length (ARL) and the Standard Deviation of the Run Length (SDRL). The proposed charts are compared with their counterparts based on SRS, RSS, and MRSS schemes. It turns out that the proposed charts are more sensitive than the existing charts.

The rest of the paper is structured in the following order: Sections 2 and 3 briefly review SEWMA and SCUSUM charts with SRS, respectively. In Section 4, the VLRSS scheme is discussed with both perfect and imperfect rankings. The proposed charts are presented in Section 5. A comparative study is conducted in Section 6. An illustrative example is presented in Section 7, and Section 8 summarizes the main findings. 


\section{The SEWMA chart}

The classical SEWMA chart is a mixture of two control charts. The Shewhart and EWMA charts provide protection against the large and small-to-moderate shifts in the process mean, respectively [cf., 5].

Let $Y$ denote the study variable and let $\left\{Y_{t}\right\}$, for $t=1,2, \cdots$, be a sequence of Independent and Identically Distributed (IID) random variables. Here, it is assumed that $Y_{t}$ is a normally distributed random variable with the in-control mean $\mu_{Y}$ and the in-control variance $\sigma_{Y}^{2}$, i.e., $Y_{t} \sim N\left(\mu_{Y}, \sigma_{Y}^{2}\right)$ for $t \geq 1$. Let $\left\{\bar{Y}_{\mathrm{SRS}, t}\right\}$ be a sequence of IID random variables with SRS, where $\bar{Y}_{\mathrm{SRS}, t}=(1 / n) \sum_{i=1}^{n} Y_{i, t}$. Here, $Y_{i, t}$ is the $i$ th observation in the $t$ th simple random sample of size $n$, for $i=1,2, \cdots, n$. Note that $\bar{Y}_{\mathrm{SRS}, t}$ is also a normal random variable with the mean $\mu_{Y}$ and the variance $\sigma_{Y}^{2} / n$, i.e., $\bar{Y}_{\mathrm{SRS}, t} \sim N\left(\mu_{Y}, \sigma_{Y}^{2} / n\right)$.

Let $\delta=\sqrt{n}\left|\mu_{Y}-\mu_{Y, 1}\right| / \sigma_{Y}$ be the amount of standardized shift to be detected in the in-control process mean $\mu_{Y}$, where $\mu_{Y, 1}$ is the out-of-control process mean.

By using $\bar{Y}_{\mathrm{SRS}, t}$, an EWMA statistic, say $Z_{t}$, is given by:

$$
Z_{t}=\lambda \bar{Y}_{\mathrm{SRS}, t}+(1-\lambda) Z_{t-1}
$$

where $Z_{t}$ and $Z_{t-1}$ are the current and past information, respectively, and $0<\lambda \leq 1$ is a smoothing constant. The starting value of $Z_{t}$ is set equal to the incontrol process mean $\mu_{Y}$, i.e., $Z_{0}=\mu_{Y}$. The variance of $Z_{t}$ is:

$$
\operatorname{Var}\left(Z_{t}\right)=\frac{\sigma_{Y}^{2}}{n} \cdot \frac{\lambda}{(2-\lambda)}\left[1-(1-\lambda)^{2 t}\right]
$$

Here, if the time $t$ gets large, the term $\left[1-(1-\lambda)^{2 t}\right]$ approaches unity. The asymptotic variance of the EWMA statistic $Z_{t}$ is given by:

$$
\operatorname{Var}\left(Z_{t}\right)=\frac{\sigma_{Y}^{2}}{n} \cdot \frac{\lambda}{(2-\lambda)}
$$

The Upper Control Limit (UCL) and the Lower Control Limit (LCL) of the EWMA chart based on the asymptotic variance of $Z_{t}$ are given by:

$$
\mathrm{UCL}=\mu_{Y}+L \frac{\sigma_{Y}}{\sqrt{n}} \sqrt{\frac{\lambda}{(2-\lambda)}},
$$

and:

$$
\mathrm{LCL}=\mu_{Y}-L \frac{\sigma_{Y}}{\sqrt{n}} \sqrt{\frac{\lambda}{(2-\lambda)}} .
$$

Similarly, the UCL and LCL of the Shewhart chart based on $\bar{Y}_{\mathrm{SRS}, t}$ are given by:

$$
\mathrm{UCL}=\mu_{Y}+d_{1} \frac{\sigma_{Y}}{\sqrt{n}},
$$

and:

$$
\mathrm{LCL}=\mu_{Y}-d_{1} \frac{\sigma_{Y}}{\sqrt{n}} .
$$

The Central Limits (CLs) of both the EWMA and Shewhart charts are set equal to the in-control process mean, i.e., $\mathrm{CL}=\mu_{Y}$. Here, $L$ and $d_{1}$ are the design parameters of the SEWMA chart, respectively, and their values depend on the choices of $\lambda$ and the desired in-control ARL. The SEWMA chart triggers an out-ofcontrol signal when either $Z_{t}$ falls outside the EWMA control limits or $\bar{Y}_{\mathrm{SRS}, t}$ falls outside the Shewhart control limits. When working with the SEWMA chart, the range of $d_{1}$ should be $3.0<d_{1}<4.5$ [cf., 5].

As mentioned earlier, the EWMA chart is very effective in detecting small-to-moderate process shifts. However, there might exist a situation where the process-initially or in the startup-may make tracks in a different direction from the process target or after the process is recouped from an out-of-control state. In such situations, giving a head-start to the EWMA chart may help earlier detection of shifts in the process target. The FIR feature in the EWMA charting structure was first suggested by Lucas and Saccucci [5] to overcome such situations. They suggested using two one-sided EWMA charts, each with a head-start. Their work was further extended by Rhoads et al. [41] who used two one-sided EWMA charts with head-starts and the time-varying control limits. To further reduce the time-varying control limits of the EWMA chart for the first few samples, say ten or twenty, Steiner [42] used an exponentially decreasing adjustment factor. The new control limits of the EWMA chart with the FIRadjustment factor are as follows:

$$
\begin{aligned}
& \mathrm{UCL}=\mu_{Y}+L \frac{\sigma_{Y}}{\sqrt{n}}\left(1-(1-f)^{1+a(t-1)}\right) \sqrt{\frac{\lambda}{(2-\lambda)}}, \\
& \mathrm{LCL}=\mu_{Y}-L \frac{\sigma_{Y}}{\sqrt{n}}\left(1-(1-f)^{1+a(t-1)}\right) \sqrt{\frac{\lambda}{(2-\lambda)}},
\end{aligned}
$$

where $f$ and $a$ are known constants. The choice of $a$, suggested by Steiner [42], for which the FIR adjustment has little effect after the 20th observation is $a=-(1 / 19)(2 / \log (1-f)+1)$. For instance, with $f=0.5$, we get $a=0.3$. For more details regarding the FIR feature with the EWMA chart, we refer to Rhoads et al. [41], Steiner [42], Knoth [6], and Haq et al. [10].

\section{The Shewhart-CUSUM chart}

SCUSUM chart was first suggested by Lucas [3], who integrated the Shewhart chart with the CUSUM chart; it is useful to detect small and large shifts in the process target simultaneously. In the SCUSUM charting structure, the CUSUM chart quickly detects small shifts, whereas the Shewhart chart swiftly detects large shifts in the process target. 
SCUSUM chart works similarly to the classical CUSUM chart. The CUSUM chart works with the two CUSUMs, upward and downward, say $C_{t}^{+}$and $C_{t}^{-}$, respectively, given by:

$$
\begin{aligned}
& C_{t}^{+}=\operatorname{Max}\left[0,+\left(\bar{Y}_{\mathrm{SRS}, t}-\mu_{Y}\right)-K+C_{t-1}^{+}\right], \\
& C_{t}^{-}=\operatorname{Max}\left[0,-\left(\bar{Y}_{\mathrm{SRS}, t}-\mu_{Y}\right)-K+C_{t-1}^{-}\right],
\end{aligned}
$$

where $K=k \sigma_{Y} / \sqrt{n}$ is the reference of slack value of the CUSUM chart. Here, $k$ is usually taken as half of the magnitude of the shift $\delta$ to be detected in $\mu_{Y}$, i.e., $k=\delta / 2$. The CUSUM chart declares the out-of-control process if either $C_{t}^{+}$or $C_{t}^{-}$exceeds the predetermined decision value $H=h \sigma_{Y} / \sqrt{n}$, where $h$ is selected to get the desired in-control ARL of the CUSUM chart. In the SCUSUM chart, the above CUSUM is integrated with the Shewhart chart, as explained in the previous section. The SCUSUM chart triggers an out-of-control signal when $C_{t}^{+}$or $C_{t}^{-}$exceeds $H$ or if $Y_{\mathrm{SRS}, t}$ exceeds the control limits given in Eq. (5) in either direction. It is customary to take $d_{1}=3.5$ [cf., 3$]$.

The FIR feature with the CUSUM chart was first suggested by Lucas and Crosier [2], which enables the CUSUM chart to react quickly against the startup/initial problems. The FIR feature in the CUSUM chart works by resetting the starting values of both plotting-CUSUMs to some non-zero constants. They recommended using $H / 2$ for a $50 \%$ head-start, i.e., by setting $C_{0}^{+}=C_{0}^{-}=H / 2$. For more details, see Lucas and Crosier [2] and Haq et al. [10].

\section{The VLRSS scheme}

In this section, we briefly review the mean estimator using VLRSS under both perfect and imperfect rankings. The VLRSS scheme is a cost-effective alternative to the SRS and RSS schemes. This scheme provides not only an unbiased and precise mean estimator when sampling from a symmetric population, but also plenty of options to the experimenter in selecting different representative samples with the less number of identified units compared to that using the RSS scheme, i.e., the ranking costs with VLRSS could be more or less than that with the RSS. It is worth mentioning that the VLRSS scheme provides a more efficient mean estimator than the mean estimators based on RSS and MRSS schemes when ranking costs are negligible. However, when ranking costs are high, it is still beneficial to use VLRSS scheme with the less ranking cost than that with the RSS schemes [cf., 18].

The main steps involved in selecting a varied $\mathrm{L}$ ranked set sample of size $n$ are presented as follows:

Step 1: Select the value of the VLRSS coefficient, say $w=[a l]$, where $0 \leq a<0.5$. Here, [.] is the largest possible integer value;
Step 2: Select $2 w l$ units from the target population. Divide these units into $2 w$ sets, with each set consisting of $l$ units;

Step 3: Rank the units within each set by any cheap or inexpensive method with respect to the study variable or using ranks of an auxiliary variable;

Step 4: Select the $v$ th and $(l-v+1)$ th smallest ranked units from the first and last $w$ sets, respectively, where $v=1,2, \cdots,[l / 2]$;

Step 5: Identify $m(m-2 w)$ units from the target population and, then, divide these units into $m-2 w$ sets, with each set comprising $m$ units;

Step 6: Select the $i$ th smallest ranked unit from the $(i+w)$ th set of $m$ units, for $i=w+1, w+2, \cdots, m-w$;

Step 7: This completes one cycle of a varied $L$ ranked set sample of size $m$. Steps 1-6 could be repeated, if necessary, $r$ number of times to get a total sample of size $n=m r$ units.

Symbolically, let $\left(Y_{i 1 j}, Y_{i 2 j}, \cdots, Y_{i l j}\right), i=1,2, \cdots, 2 w$, be $2 w$ samples, each of size $l$, for the $j$ th cycle, where $j=1,2, \cdots, r$. Let $Y_{i(v: l) j}$ denote the $v$ th order statistic of $\left(Y_{i 1 j}, Y_{i 2 j}, \cdots, Y_{i l j}\right)$ for $i=1,2, \cdots, w$, and let $Y_{i(l-v+1: l) j}$ be the $(l-v+1)$ th order statistic of $\left(Y_{i 1 j}, Y_{i 2 j}, \cdots, Y_{i l j}\right)$ for $i=w+1, w+2, \cdots, 2 w$. Let $\left(Y_{(i+w) 1 j}, Y_{(i+w) 2 j}, \cdots, Y_{(i+w) m j}\right), i=w+1,2, \cdots, m-$ $w$, denote $m-2 w$ samples, each of size $m$, for the $j$ th cycle. Let $Y_{i+w(i: m) j}$ denote the $i$ th order statistic of $\left(Y_{(i+w) 1 j}, Y_{(i+w) 2 j}, \cdots, Y_{(i+w) m j}\right)$ for $i=1,2, \cdots, m-$ $w$.

The sample mean based on a varied $\mathrm{L}$ ranked set sample of size $n$, denoted by $\bar{Y}_{\text {VLRSS }}$, and its variance, respectively, are given by:

$$
\begin{gathered}
\bar{Y}_{\text {VLRSS }}=\frac{1}{n} \sum_{j=1}^{r}\left(\sum_{i=1}^{w} Y_{i(v: l) j}+\sum_{i=w+1}^{2 w} Y_{i(l-v+1: l) j}\right. \\
\left.+\sum_{i=w+1}^{m-w} Y_{i+w(i: m) j}\right), \\
\operatorname{Var}\left(\bar{Y}_{\text {VLRSS }}\right)=\frac{1}{n m}\left(w\left(\sigma_{Y(v: l)}^{2}+\sigma_{Y(l-v+1: l)}^{2}\right)\right. \\
\left.+\sum_{i=w+1}^{m-w} \sigma_{Y(i: m)}^{2}\right),
\end{gathered}
$$

where:

$$
\begin{aligned}
& \sigma_{Y(v: l)}^{2}=\operatorname{Var}\left(Y_{i(v: l) j}\right), \\
& \sigma_{Y(l-v+1: l)}^{2}=\operatorname{Var}\left(Y_{i(l-v+1: l) j}\right), \text { and } \\
& \sigma_{Y(i: m)}^{2}=\operatorname{Var}\left(Y_{i(i: m) j}\right) .
\end{aligned}
$$

For more details regarding the computation of the 
variances of order statistics, we refer to David and Nagaraja [43].

For a symmetric population, Haq et al. [18] showed that $\bar{Y}_{\text {VLRSS }}$ is an unbiased estimator of $\mu_{Y}$. They have also shown that, with some suitable choices of $v, l$, and $w$, the existing RSS schemes are special cases of VLRSS. For instance, for $w=0$, VLRSS becomes RSS; for $w=[(m-1) / 2], l=m$, and $v=w+1$, VLRSS becomes MRSS, etc. While selecting a varied $\mathrm{L}$ ranked set sample of size $n$, the experimenter needs to identify $n m-2 w(m-l) r$ units, while the classical RSS and MRSS require identifying $n \mathrm{~m}$ units when selecting a sample of size $n$. It is to be noted that when $m>l$, VLRSS requires fewer identified units than that when using the RSS or MRSS [cf., $18]$.

\subsection{The imperfect VLRSS scheme}

There may exist a situation where it is not possible to rank the study variable visually, or it is costly and time consuming. This issue can be solved by ranking the study variable $(Y)$ using the ranks of a highly correlated variable, say $X$, given that it is readily available. Stokes [13] suggested a simple model for the imperfect rankings, given by

$$
\begin{gathered}
Y_{i[i: u] j}=\mu_{Y}+\rho \frac{\sigma_{Y}}{\sigma_{X}}\left(X_{i(i: u) j}-\mu_{X}\right)+\xi_{i j}, \\
i=1,2, \cdots, u, \quad j=1,2, \cdots, r,
\end{gathered}
$$

where $u=l, m ; \mu_{X}$ and $\sigma_{X}$ are the population mean and standard deviation of $X$, respectively, and $\rho$ is the correlation between $Y$ and $X$. Here, $\xi_{i j} \sim N\left(0, \sigma_{Y}^{2}(1-\right.$ $\left.\rho^{2}\right)$ ), and $X_{i(i: u) j}$ and $\xi_{i j}$ are mutually independent. $Y_{i[i: u] j}$ is the $i$ th concomitant or induced order statistic corresponding to the $i$ th order statistic $X_{i(i: u) j}, i=$ $1,2, \cdots, u$. The values of $X$ are perfectly ranked; however, those of $Y$ are ranked with error. On the lines of Stokes [13], the sample mean under Imperfect VLRSS (IVLRSS), say $\bar{Y}_{\text {IVLRSS }}$, and its variance, are, respectively, given by:

$$
\begin{aligned}
\bar{Y}_{\text {IVLRSS }}= & \frac{1}{n} \sum_{j=1}^{r}\left(\sum_{i=1}^{w} Y_{i[v: l] j}+\sum_{i=w+1}^{2 w} Y_{i[l-v+1: l] j}\right. \\
& \left.+\sum_{i=w+1}^{m-w} Y_{i+w[i: m] j}\right)
\end{aligned}
$$

and:

$$
\begin{aligned}
\operatorname{Var}\left(\bar{Y}_{\mathrm{VLRSS}}\right)= & \frac{1}{n m}\left(w\left(\sigma_{Y[v: l]}^{2}+\sigma_{Y[l-v+1: l]}^{2}\right)\right. \\
& \left.+\sum_{i=w+1}^{m-w} \sigma_{Y[i: m]}^{2}\right)
\end{aligned}
$$

$$
\begin{aligned}
= & \frac{1}{n m}\left\{m \sigma_{Y}^{2}\left(1-\rho^{2}\right)+\rho^{2} \frac{\sigma_{Y}^{2}}{\sigma_{X}^{2}}\left(2 w \sigma_{X(v: l)}^{2}\right.\right. \\
& \left.\left.+\sum_{i=w+1}^{m-w} \sigma_{X(i: m)}^{2}\right)\right\}
\end{aligned}
$$

where:

$$
\begin{aligned}
& \sigma_{Y[v: l]}^{2}=\operatorname{Var}\left(Y_{i[v: l] j}\right), \\
& \sigma_{Y[l-v+1: l]}^{2}=\operatorname{Var}\left(Y_{i[l-v+1: l] j}\right), \text { and } \\
& \sigma_{Y[i: m]}^{2}=\operatorname{Var}\left(Y_{i[i: m] j}\right) .
\end{aligned}
$$

For more details on the computation of these variances, we refer to see David and Nagaraja [43]. When sampling from a symmetric bivariate population, $\bar{Y}_{\text {IVLRSS }}$ is unbiased, is characterized by reasonable assumptions, and is more precise than the mean estimators based on imperfect RSS and MRSS schemes [cf., 18].

\section{The proposed control charts}

In this section, new SEWMA and SCUSUM control charts are proposed for efficiently monitoring the process mean $\mu_{Y}$ under both perfect and imperfect VLRSS schemes. The run length characteristics of these control charts are also computed through Monte Carlo simulations.

\subsection{The SEWMA chart}

Suppose that a sample of size $n$ is selected from the target population with $\mathrm{S}$ scheme at the time $t(\geq 1)$, where $\mathrm{S}=$ VLRSS and IVLRSS. Let $\left\{\bar{Y}_{\mathrm{S}, t}\right\}$ be a sequence of IID random variables for $t=1,2, \cdots$. By considering $\left\{\bar{Y}_{\mathrm{S}, t}\right\}$, it is possible to construct an SEWMA chart for monitoring $\mu_{Y}$. The plottingstatistic of the SEWMA chart with $\mathrm{S}$ scheme is given by:

$$
Q_{t}=\lambda \bar{Y}_{\mathrm{S}, t}+(1-\lambda) Q_{t-1},
$$

where $\lambda$ is a smoothing constant. The asymptotic variance of $Q_{t}$ is:

$$
\operatorname{Var}\left(Q_{t}\right)=\operatorname{Var}\left(\bar{Y}_{\mathrm{S}, t}\right) \cdot \frac{\lambda}{(2-\lambda)},
$$

where $\operatorname{Var}\left(\bar{Y}_{\mathrm{S}, t}\right)$ denotes the variance of $\left\{\bar{Y}_{\mathrm{S}, t}\right\}$ at the time $t$. The control limits of the SEWMA chart with S scheme are:

$$
\mathrm{UCL}=\mu_{Y}+L \sqrt{\operatorname{Var}\left(\bar{Y}_{\mathrm{S}, t}\right)} \sqrt{\frac{\lambda}{(2-\lambda)}},
$$

and: 


$$
\mathrm{LCL}=\mu_{Y}-L \sqrt{\operatorname{Var}\left(\bar{Y}_{\mathrm{S}, t}\right)} \sqrt{\frac{\lambda}{(2-\lambda)}} .
$$

Similarly, the control limits of the Shewhart chart, based on $\left\{\bar{Y}_{\mathrm{S}, t}\right\}$, are given by:

$$
\mathrm{UCL}=\mu_{Y}+d_{1} \sqrt{\operatorname{Var}\left(\bar{Y}_{\mathrm{S}, t}\right)}
$$

and:

$$
\mathrm{LCL}=\mu_{Y}-d_{1} \sqrt{\operatorname{Var}\left(\bar{Y}_{\mathrm{S}, t}\right)},
$$

where $L$ and $d_{1}$ are positive control charting multipliers that are selected to ensure that the in-control ARL of the SEWMA chart has reached a certain level. The SEWMA chart triggers an out-of-control signal whenever $Q_{t}$ or $\left\{\bar{Y}_{\mathrm{S}, t}\right\}$ falls outside their respective control limits' intervals, i.e., [LCL, UCL]. As mentioned earlier, the sensitivity of the SEWMA chart can be enhanced by giving a head-start to the SEWMA chart with the FIR-adjustment [cf., 42]; on the similar lines, an FIR feature is associated with the proposed SEWMA chart by setting $f=0.5$ and $a=0.3$, as recommended by Steiner [42].

\subsection{The SCUSUM chart}

To construct an SCUSUM chart for monitoring $\mu_{Y}$, consider the sequence $\left\{\bar{Y}_{\mathrm{S}, t}\right\}$ for $t=1,2, \cdots$. The plotting-statistics (upper and lower CUSUMs) of the proposed SCUSUM chart using S scheme are, respectively, defined by:

$$
\begin{aligned}
& C_{t}^{+}=\operatorname{Max}\left[0,+\left(\bar{Y}_{\mathrm{S}, t}-\mu_{Y}\right)-K+C_{t-1}^{+}\right], \\
& C_{t}^{-}=\operatorname{Max}\left[0,-\left(\bar{Y}_{\mathrm{S}, t}-\mu_{Y}\right)-K+C_{t-1}^{-}\right],
\end{aligned}
$$

where $C_{0}^{+}=C_{0}^{-}=0$. The reference value, $K$, and the decision interval, $H$, of the SCUSUM chart are respectively as follows:

$$
\begin{aligned}
& K=k \sqrt{\operatorname{Var}\left(\bar{Y}_{\mathrm{S}, t}\right)}, \\
& H=h \sqrt{\operatorname{Var}\left(\bar{Y}_{\mathrm{S}, t}\right)},
\end{aligned}
$$

where the values of $k$ and $h$ are the same as explained in the previous section. Similarly, the control limits of the Shewhart chart based on $\left\{\bar{Y}_{\mathrm{S}, t}\right\}$ are given by:

$$
\mathrm{UCL}=\mu_{Y}+d_{1} \sqrt{\operatorname{Var}\left(\bar{Y}_{\mathrm{S}, t}\right)},
$$

and:

$$
\mathrm{LCL}=\mu_{Y}-d_{1} \sqrt{\operatorname{Var}\left(\bar{Y}_{\mathrm{S}, t}\right)}
$$

The SCUSUM triggers an out-of-control signal if $C_{t}^{+}$ or $C_{t}^{-}$exceeds $H$ or if $\bar{Y}_{\mathrm{S}, t}$ is less than LCL or greater than UCL of the Shewhart chart. The sensitivity of the CUSUM chart for the start-up problems, as suggested by Lucas and Crosier [2], could increase with a headstart feature. They recommended resetting the starting values of $C_{t}^{+}$and $C_{t}^{-}$to non-zero constants, like $C_{0}^{+}=$ $C_{0}^{-}=H / 2$ for an $50 \%$ head-start [cf., 2]. On the same lines, an FIR feature is attached to the SCUSUM chart with $50 \%$ head-start.

\subsection{Run length evaluation}

Generally, the run length performance of a control chart is evaluated in terms of its run length characteristics including the ARL and the SDRL. For an in-control process, the in-control ARL should be large enough to avoid false alarms, while, for an out-of-control process, it should be as small as possible so that the control chart can swiftly trigger an out-of-control signal. In the literature, there exist some methods that could be used to compute the run length characteristics of a control chart, including the integral equations, Markov chain, and the Monte Carlo simulations. The Monte Carlo simulation method is broadly used to compute the run length characteristics of the control charts, and thus it is used here.

In order to evaluate the run length performances of the proposed control charts, we generate samples under VLRSS from a normal distribution. The incontrol ARL is set equal to 500- a choice recommended by the SPC practitioners. Here, each simulation run comprises 50,000 iterations of the run length. In Tables 1 and 2 , the values of $(\lambda, L)$ and $(k, h)$ are reported for the SEWMA and SCUSUM charts, respectively, with different possible values of $(m, l, v)$ with $r=1$ when the in-control ARL is matched as 500. These constants could be used when using the proposed charts with different choices of $(m, l, v)$ when the in-control ARL is fixed to 500 .

For brevity of discussion, without loss of generality, with $n=5$ and $r=1$, using different pairs of $(l, v)$ with $w=2$, we compute the ARLs and SDRLs of the proposed control charts in Tables 3-6 (with and without FIR features). It is to be noted that, for a given sample size $n$, we consider those choices of $w$ and $(l, v)$ with the VLRSS scheme for which the mean estimator is precise [cf., 18]. Different values of $\delta$ are considered, i.e., $\delta=0(0.25) 4$. For both the SEWMA and SCUSUM charts, different values of $\lambda$ and $k$ have been considered. The values of $d_{1}$ for the SEWMA and SCUSUM charts are set equal to 3.31 and 3.50, respectively. Moreover, the results are computed when sampling from a normal distribution. Here, under each simulation run, 50,000 replications of the run length are considered. It is observed that the out-of-control ARLs tend to decrease as the value of $\delta$ increases, and vice versa. A similar trend is observed when a control chart is constructed with the FIR feature. 
Table 1. The values of $\mathrm{L}$ with different choices of $(l, v)$ when the in-control ARL of the SEWMA-VLRSS chart is 500.

\begin{tabular}{|c|c|c|c|c|c|c|c|c|}
\hline \multirow[b]{2}{*}{$\lambda$} & \multirow{2}{*}{$\begin{array}{c}m=2 \\
(0,0)\end{array}$} & & \multicolumn{6}{|c|}{$m=4$} \\
\hline & & & $(2,1)$ & $(3,2)$ & $(4,1)$ & $(4,2)$ & $(5,3)$ & $(6,3)$ \\
\hline 0.05 & 2.2582 & & 2.8510 & 2.8523 & 2.8800 & 2.8490 & 2.8492 & 2.8485 \\
\hline 0.10 & 2.4260 & & 3.0270 & 3.0164 & 3.0463 & 3.0257 & 3.0225 & 3.0230 \\
\hline 0.25 & 2.5789 & & 3.1700 & 3.1666 & 3.1856 & 3.1650 & 3.1659 & 3.1648 \\
\hline 0.50 & 2.6350 & & 3.1755 & 3.1700 & 3.1889 & 3.1747 & 3.1695 & 3.1755 \\
\hline 0.75 & 2.6470 & & 3.1230 & 3.1186 & 3.1390 & 3.1190 & 3.1155 & 3.1161 \\
\hline \multirow[t]{2}{*}{1.00} & 2.6465 & & 3.1050 & 3.1000 & 3.1158 & 3.0990 & 3.1000 & 3.1000 \\
\hline & \multicolumn{8}{|c|}{$m=3, w=1$} \\
\hline$\lambda$ & $(2,1)$ & $(3,1)$ & $(3,2)$ & $(4,1)$ & $(4,2)$ & $(5,1)$ & $(5,2)$ & $(5,3)$ \\
\hline 0.05 & 2.8600 & 2.8800 & 2.8519 & 2.8890 & 2.8510 & 2.8990 & 2.8585 & 2.8522 \\
\hline 0.10 & 3.0307 & 3.0469 & 3.0285 & 3.0599 & 3.0285 & 3.0690 & 3.0308 & 3.0290 \\
\hline 0.25 & 3.1730 & 3.1860 & 3.1710 & 3.2004 & 3.1710 & 3.2100 & 3.1683 & 3.1660 \\
\hline 0.50 & 3.1763 & 3.1921 & 3.1758 & 3.2056 & 3.1759 & 3.2105 & 3.1800 & 3.1759 \\
\hline 0.75 & 3.1240 & 3.1377 & 3.1200 & 3.1499 & 3.1207 & 3.1545 & 3.1230 & 3.1203 \\
\hline \multirow[t]{3}{*}{1.00} & 3.1070 & 3.1157 & 3.1025 & 3.1260 & 3.1028 & 3.1313 & 3.1070 & 3.1013 \\
\hline & \multicolumn{8}{|c|}{$m=5$} \\
\hline & \multicolumn{4}{|c|}{$w=1$} & \multicolumn{4}{|c|}{$w=2$} \\
\hline$\lambda$ & $(2,1)$ & $(3,2)$ & $(4,2)$ & $(5,1)$ & $(5,3)$ & $(6,3)$ & $(7,4)$ & $(8,4)$ \\
\hline 0.05 & 2.8494 & 2.8490 & 2.8480 & 2.8740 & 2.8450 & 2.8460 & 2.8444 & 2.8448 \\
\hline 0.10 & 3.0270 & 3.0190 & 3.0250 & 3.0450 & 3.0170 & 3.0179 & 3.0164 & 3.0164 \\
\hline 0.25 & 3.1695 & 3.1654 & 3.1645 & 3.1850 & 3.1570 & 3.1579 & 3.1584 & 3.1590 \\
\hline 0.50 & 3.1747 & 3.1690 & 3.1755 & 3.1920 & 3.1660 & 3.1669 & 3.1670 & 3.1673 \\
\hline 0.75 & 3.1195 & 3.1150 & 3.1150 & 3.1370 & 3.1150 & 3.1150 & 3.1134 & 3.1155 \\
\hline 1.00 & 3.1000 & 3.1000 & 3.1000 & 3.1147 & 3.0980 & 3.0968 & 3.0945 & 3.0970 \\
\hline
\end{tabular}

Table 2. The values of $h$ with different choices of $(l, v)$ when the in-control ARL of the SCUSUM-VLRSS chart is 500.

\begin{tabular}{|c|c|c|c|c|c|c|c|c|}
\hline \multirow[b]{2}{*}{$k$} & \multirow{2}{*}{$\begin{array}{c}m=2 \\
(0,0)\end{array}$} & & \multicolumn{6}{|c|}{$m=4, w=1$} \\
\hline & & & $(2,1)$ & $(3,2)$ & $(4,1)$ & $(4,2)$ & $(5,3)$ & $(6,3)$ \\
\hline 0.25 & 9.1100 & & 9.0950 & 9.0740 & 9.1100 & 9.0970 & 9.0760 & 9.0875 \\
\hline 0.50 & 5.3280 & & 5.3166 & 5.3105 & 5.3360 & 5.3050 & 5.3050 & 5.3077 \\
\hline 0.75 & 3.6980 & & 3.6785 & 3.6765 & 3.6950 & 3.6753 & 3.6758 & 3.6760 \\
\hline \multirow[t]{2}{*}{1.00} & 2.7450 & & 2.7282 & 2.7255 & 2.7440 & 2.7245 & 2.7240 & 2.7252 \\
\hline & \multicolumn{8}{|c|}{$m=3, w=1$} \\
\hline$k$ & $(2,1)$ & $(3,1)$ & $(3,2)$ & $(4,1)$ & $(4,2)$ & $(5,1)$ & $(5,2)$ & $(5,3)$ \\
\hline 0.25 & 9.0953 & 9.1109 & 9.0700 & 9.1230 & 9.0950 & 9.1560 & 9.0950 & 9.0747 \\
\hline 0.50 & 5.3164 & 5.3340 & 5.3133 & 5.3430 & 5.3085 & 5.3780 & 5.3143 & 5.3060 \\
\hline 0.75 & 3.6791 & 3.6980 & 3.6767 & 3.7122 & 3.6790 & 3.7181 & 3.6800 & 3.6767 \\
\hline \multirow[t]{3}{*}{1.00} & 2.7320 & 2.7450 & 2.7280 & 2.7547 & 2.7284 & 2.7670 & 2.7290 & 2.7287 \\
\hline & \multicolumn{8}{|c|}{$m=5$} \\
\hline & \multicolumn{4}{|c|}{$w=1$} & \multicolumn{4}{|c|}{$w=2$} \\
\hline$k$ & $(2,1)$ & $(3,2)$ & $(4,2)$ & $(5,1)$ & $(5,3)$ & $(6,3)$ & $(7,4)$ & $(8,4)$ \\
\hline 0.25 & 9.0959 & 9.0739 & 9.0755 & 9.1110 & 9.0490 & 9.0499 & 9.0508 & 9.0510 \\
\hline 0.50 & 5.3109 & 5.3080 & 5.3080 & 5.3270 & 5.2930 & 5.2935 & 5.2938 & 5.2938 \\
\hline 0.75 & 3.6760 & 3.6755 & 3.6746 & 3.6960 & 3.6670 & 3.6682 & 3.6666 & 3.6682 \\
\hline 1.00 & 2.7250 & 2.7245 & 2.7260 & 2.7460 & 2.7170 & 2.7193 & 2.7185 & 2.7186 \\
\hline
\end{tabular}

\subsection{When the process parameters are unknown}

If the underlying process parameters are not known in advance-phase-I monitoring, then it is customary to estimate them using a large historical dataset, provided that it has been obtained from an in-control process. Suppose that, from an in-control process, $q$ subgroups and each of size $m$ are available with $\mathrm{S}$ scheme.

In the perfect ranking case, $\mu_{Y}$ and $\operatorname{Var}\left(\bar{Y}_{\text {VLRSS }}\right)$ could be estimated by using their respective unbiased 
Table 3. The run length profiles of SEWMA-VLRSS chart when the in-control ARL is 500 .

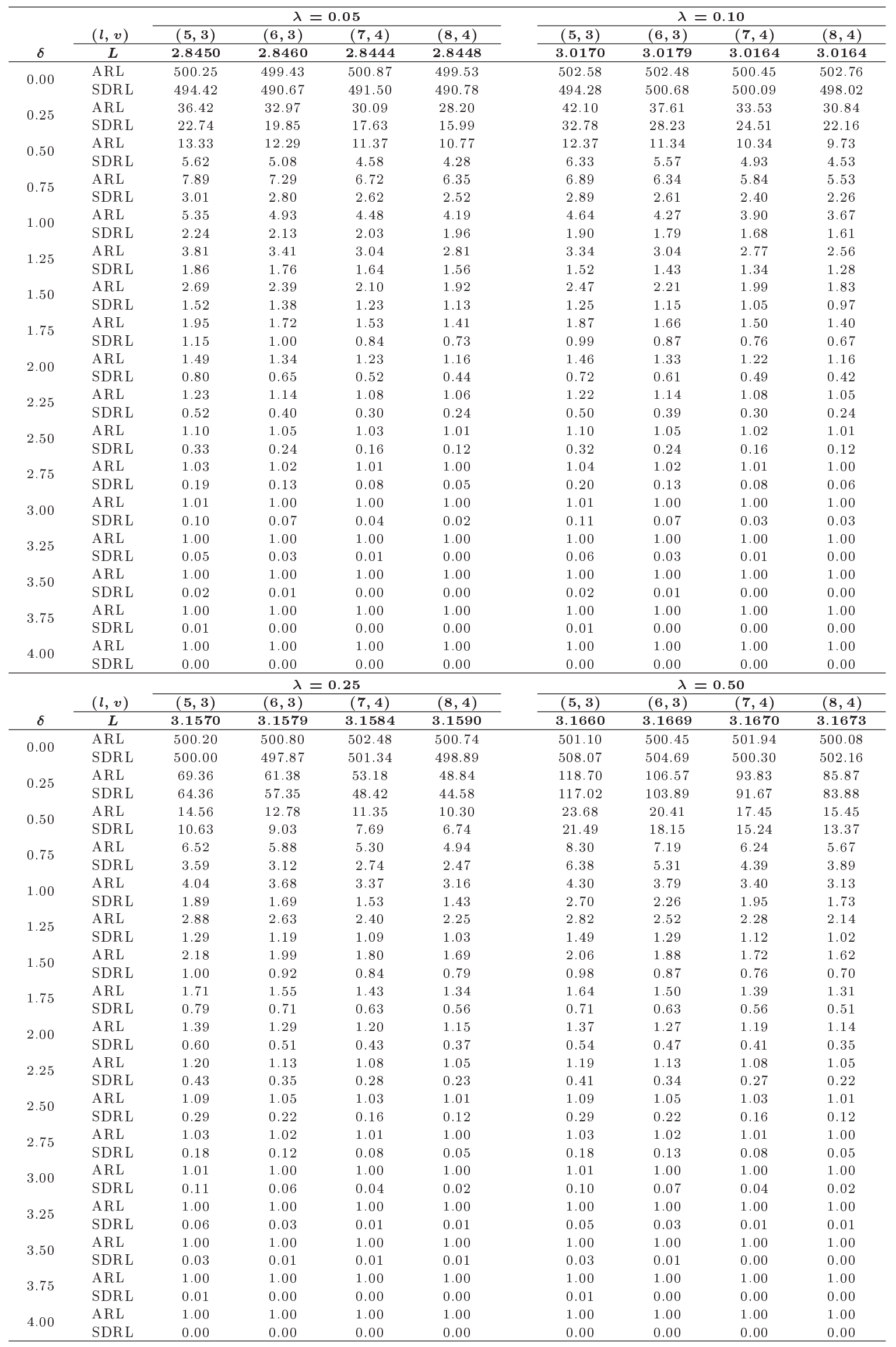


Table 4. The run length profiles of SCUSUM-VLRSS chart when the in-control ARL is 500.

\begin{tabular}{|c|c|c|c|c|c|c|c|c|c|}
\hline \multirow[b]{3}{*}{$\delta$} & \multirow[b]{2}{*}{$(l, \boldsymbol{v})$} & \multicolumn{4}{|c|}{$k=0.25$} & \multicolumn{4}{|c|}{$k=0.50$} \\
\hline & & $(5,3)$ & $(6,3)$ & $(7,4)$ & $(8,4)$ & $(5,3)$ & $(6,3)$ & $(7,4)$ & $(8,4)$ \\
\hline & $h$ & 9.0490 & 9.0499 & 9.0508 & 9.0510 & 5.2930 & 5.2935 & 5.2938 & 5.2938 \\
\hline O & ARL & 500.80 & 500.62 & 500.72 & 500.26 & 502.65 & 500.08 & 501.11 & 499.16 \\
\hline 0.00 & SDRL & 486.65 & 490.59 & 492.92 & 487.82 & 497.32 & 493.26 & 494.90 & 491.04 \\
\hline $0 \cdot 25$ & ARL & 36.00 & 32.87 & 30.02 & 28.32 & 47.16 & 41.55 & 36.91 & 33.68 \\
\hline 0.20 & SDRL & 21.62 & 18.97 & 16.75 & 15.31 & 39.74 & 34.25 & 29.62 & 26.48 \\
\hline 0.50 & ARL & 13.64 & 12.66 & 11.76 & 11.17 & 12.17 & 11.00 & 9.96 & 9.32 \\
\hline 0.00 & SDRL & 5.36 & 4.82 & 4.36 & 4.09 & 6.73 & 5.83 & 5.06 & 4.60 \\
\hline 075 & ARL & 8.25 & 7.65 & 7.11 & 6.73 & 6.48 & 5.95 & 5.50 & 5.17 \\
\hline 0 & SDRL & 2.88 & 2.66 & 2.49 & 2.38 & 2.76 & 2.44 & 2.22 & 2.06 \\
\hline 100 & ARL & 5.71 & 5.27 & 4.85 & 4.54 & 4.37 & 4.05 & 3.73 & 3.52 \\
\hline 1.00 & SDRL & 2.11 & 2.02 & 1.94 & 1.88 & 1.71 & 1.56 & 1.44 & 1.37 \\
\hline 1.25 & ARL & 4.15 & 3.76 & 3.38 & 3.12 & 3.23 & 2.97 & 2.72 & 2.55 \\
\hline 1.20 & SDRL & 1.81 & 1.73 & 1.65 & 1.58 & 1.28 & 1.21 & 1.14 & 1.09 \\
\hline 1.50 & ARL & 3.02 & 2.69 & 2.37 & 2.16 & 2.48 & 2.26 & 2.05 & 1.91 \\
\hline 1.00 & SDRL & 1.56 & 1.45 & 1.33 & 1.24 & 1.08 & 1.01 & 0.95 & 0.90 \\
\hline 175 & ARL & 2.18 & 1.92 & 1.69 & 1.55 & 1.94 & 1.75 & 1.58 & 1.48 \\
\hline 1.10 & SDRL & 1.25 & 1.11 & 0.96 & 0.85 & 0.91 & 0.84 & 0.75 & 0.69 \\
\hline 200 & ARL & 1.65 & 1.46 & 1.31 & 1.23 & 1.54 & 1.40 & 1.28 & 1.21 \\
\hline 2.00 & SDRL & 0.92 & 0.77 & 0.62 & 0.51 & 0.73 & 0.64 & 0.53 & 0.46 \\
\hline 2.25 & ARL & 1.31 & 1.20 & 1.12 & 1.08 & 1.28 & 1.19 & 1.12 & 1.08 \\
\hline 2.20 & SDRL & 0.62 & 0.49 & 0.36 & 0.30 & 0.54 & 0.43 & 0.34 & 0.28 \\
\hline 2,50 & ARL & 1.14 & 1.08 & 1.04 & 1.02 & 1.13 & 1.08 & 1.04 & 1.02 \\
\hline 2.00 & SDRL & 0.39 & 0.29 & 0.20 & 0.16 & 0.36 & 0.28 & 0.20 & 0.15 \\
\hline 275 & ARL & 1.05 & 1.03 & 1.01 & 1.01 & 1.05 & 1.03 & 1.01 & 1.01 \\
\hline 2.10 & SDRL & 0.24 & 0.16 & 0.11 & 0.07 & 0.23 & 0.16 & 0.11 & 0.08 \\
\hline 3.00 & ARL & 1.02 & 1.01 & 1.00 & 1.00 & 1.02 & 1.01 & 1.00 & 1.00 \\
\hline .00 & SDRL & 0.13 & 0.09 & 0.04 & 0.04 & 0.13 & 0.09 & 0.05 & 0.04 \\
\hline 3.25 & ARL & 1.01 & 1.00 & 1.00 & 1.00 & 1.01 & 1.00 & 1.00 & 1.00 \\
\hline 5.20 & SDRL & 0.07 & 0.04 & 0.02 & 0.01 & 0.07 & 0.04 & 0.02 & 0.01 \\
\hline 3.50 & ARL & 1.00 & 1.00 & 1.00 & 1.00 & 1.00 & 1.00 & 1.00 & 1.00 \\
\hline 5.000 & SDRL & 0.03 & 0.02 & 0.01 & 0.00 & 0.04 & 0.02 & 0.01 & 0.00 \\
\hline 3.75 & ARL & 1.00 & 1.00 & 1.00 & 1.00 & 1.00 & 1.00 & 1.00 & 1.00 \\
\hline ש. & SDRL & 0.02 & 0.01 & 0.00 & 0.00 & 0.01 & 0.01 & 0.00 & 0.00 \\
\hline 4.00 & ARL & 1.00 & 1.00 & 1.00 & 1.00 & 1.00 & 1.00 & 1.00 & 1.00 \\
\hline 1.00 & SDRL & 0.00 & 0.00 & 0.00 & 0.00 & 0.00 & 0.01 & 0.00 & 0.00 \\
\hline & & & & 75 & & & $k=$ & .00 & \\
\hline & $(l, v)$ & $(5,3)$ & $(6,3)$ & $(7,4)$ & $(8,4)$ & $(5,3)$ & $(6,3)$ & $(7,4)$ & $(8,4)$ \\
\hline$\delta$ & $h$ & 3.6670 & 3.6682 & 3.6666 & 3.6682 & 2.7170 & 2.7193 & 2.7185 & 2.7186 \\
\hline 0.00 & ARL & 502.85 & 502.68 & 502.63 & 500.05 & 500.36 & 499.33 & 501.55 & 500.13 \\
\hline 0.00 & SDRL & 499.40 & 497.36 & 501.27 & 495.78 & 497.91 & 496.82 & 500.44 & 498.09 \\
\hline ( 25 & ARL & 70.37 & 61.78 & 53.89 & 48.85 & 96.77 & 86.37 & 75.50 & 68.85 \\
\hline 0.20 & SDRL & 66.21 & 57.04 & 49.40 & 44.55 & 94.02 & 84.06 & 72.87 & 66.29 \\
\hline 0.50 & ARL & 13.86 & 12.16 & 10.70 & 9.81 & 17.85 & 15.30 & 13.16 & 11.72 \\
\hline 0.00 & SDRL & 10.04 & 8.45 & 7.10 & 6.29 & 15.22 & 12.52 & 10.48 & 9.08 \\
\hline ח & ARL & 502.85 & 502.68 & 502.63 & 500.05 & 500.36 & 499.33 & 501.55 & 500.13 \\
\hline 0.00 & SDRL & 499.40 & 497.36 & 501.27 & 495.78 & 497.91 & 496.82 & 500.44 & 498.09 \\
\hline 0.25 & ARL & 70.37 & 61.78 & 53.89 & 48.85 & 96.77 & 86.37 & 75.50 & 68.85 \\
\hline 0.20 & SDRL & 66.21 & 57.04 & 49.40 & 44.55 & 94.02 & 84.06 & 72.87 & 66.29 \\
\hline 0.50 & ARL & 13.86 & 12.16 & 10.70 & 9.81 & 17.85 & 15.30 & 13.16 & 11.72 \\
\hline 0.00 & SDRL & 10.04 & 8.45 & 7.10 & 6.29 & 15.22 & 12.52 & 10.48 & 9.08 \\
\hline 075 & ARL & 6.23 & 5.60 & 5.11 & 4.73 & 6.69 & 5.91 & 5.26 & 4.81 \\
\hline 0.10 & SDRL & 3.32 & 2.85 & 2.51 & 2.25 & 4.378 & 3.68 & 3.13 & 2.76 \\
\hline 1.00 & ARL & 3.92 & 3.59 & 3.28 & 3.09 & 3.84 & 3.48 & 3.16 & 2.96 \\
\hline 1.00 & SDRL & 1.75 & 1.55 & 1.38 & 1.30 & 1.99 & 1.71 & 1.49 & 1.36 \\
\hline 1.25 & ARL & 2.83 & 2.61 & 2.40 & 2.25 & 2.68 & 2.46 & 2.27 & 2.13 \\
\hline 1.20 & SDRL & 1.17 & 1.07 & 0.99 & 0.93 & 1.18 & 1.05 & 0.95 & 0.87 \\
\hline 1.50 & ARL & 2.19 & 2.01 & 1.84 & 1.74 & 2.08 & 1.92 & 1.77 & 1.67 \\
\hline 1.000 & SDRL & 0.90 & 0.84 & 0.77 & 0.73 & 0.84 & 0.76 & 0.69 & 0.65 \\
\hline 175 & ARL & 1.76 & 1.61 & 1.48 & 1.40 & 1.69 & 1.57 & 1.46 & 1.38 \\
\hline 1.10 & SDRL & 0.73 & 0.67 & 0.60 & 0.56 & 0.66 & 0.61 & 0.56 & 0.52 \\
\hline 200 & ARL & 1.45 & 1.34 & 1.25 & 1.19 & 1.44 & 1.33 & 1.24 & 1.19 \\
\hline 2.00 & SDRL & 0.58 & 0.52 & 0.46 & 0.40 & 0.55 & 0.49 & 0.44 & 0.40 \\
\hline 225 & ARL & 1.25 & 1.17 & 1.11 & 1.07 & 1.24 & 1.17 & 1.11 & 1.07 \\
\hline 2.20 & SDRL & 0.45 & 0.39 & 0.32 & 0.26 & 0.44 & 0.38 & 0.31 & 0.26 \\
\hline 250 & ARL & 1.12 & 1.07 & 1.04 & 1.02 & 1.12 & 1.07 & 1.04 & 1.02 \\
\hline 2.00 & SDRL & 0.33 & 0.26 & 0.20 & 0.15 & 0.33 & 0.26 & 0.20 & 0.15 \\
\hline 275 & ARL & 1.05 & 1.02 & 1.01 & 1.01 & 1.05 & 1.03 & 1.01 & 1.01 \\
\hline 2.10 & SDRL & 0.22 & 0.15 & 0.10 & 0.08 & 0.22 & 0.16 & 0.10 & 0.07 \\
\hline 3.00 & ARL & 1.02 & 1.01 & 1.00 & 1.00 & 1.02 & 1.01 & 1.00 & 1.00 \\
\hline & SDRL & 0.13 & 0.08 & 0.05 & 0.03 & 0.13 & 0.09 & 0.04 & 0.03 \\
\hline 3.25 & ARL & 1.00 & 1.00 & 1.00 & 1.00 & 1.01 & 1.00 & 1.00 & 1.00 \\
\hline & SDRL & 0.07 & 0.04 & 0.02 & 0.01 & 0.08 & 0.04 & 0.02 & 0.01 \\
\hline 3.50 & ARL & 1.00 & 1.00 & 1.00 & 1.00 & 1.00 & 1.00 & 1.00 & 1.00 \\
\hline 0.00 & SDRL & 0.04 & 0.02 & 0.00 & 0.00 & 0.04 & 0.01 & 0.01 & 0.00 \\
\hline 375 & ARL & 1.00 & 1.00 & 1.00 & 1.00 & 1.00 & 1.00 & 1.00 & 1.00 \\
\hline & SDRL & 0.02 & 0.00 & 0.00 & 0.00 & 0.01 & 0.00 & 0.00 & 0.00 \\
\hline 4.00 & ARL & 1.00 & 1.00 & 1.00 & 1.00 & 1.00 & 1.00 & 1.00 & 1.00 \\
\hline 4.00 & SDRL & 0.00 & 0.00 & 0.00 & 0.00 & 0.01 & 0.00 & 0.00 & 0.00 \\
\hline
\end{tabular}


Table 5. The run length profiles of SEWMA-VLRSS chart with the FIR feature for the time when the in-control ARL is 500.

\begin{tabular}{|c|c|c|c|c|c|c|c|c|c|}
\hline$\delta$ & $(l, v)$ & \multicolumn{4}{|c|}{$\lambda=0.05$} & \multicolumn{4}{|c|}{$\lambda=0.10$} \\
\hline 0.00 & ARL & 499.65 & 501.08 & 502.93 & 500.20 & 501.52 & 501.09 & 502.12 & 500.81 \\
\hline 0.25 & ARL & 35.57 & 32.18 & 29.27 & 27.25 & 39.47 & 34.99 & 30.98 & 28.24 \\
\hline 0.20 & SDRL & 23.10 & 20.41 & 17.88 & 16.22 & 33.06 & 28.62 & 24.90 & 22.39 \\
\hline 0.50 & ARL & 12.07 & 11.06 & 10.06 & 9.46 & 10.28 & 9.22 & 8.29 & 7.70 \\
\hline 0.50 & SDRL & 5.93 & 5.39 & 4.87 & 4.51 & 6.59 & 5.79 & 5.08 & 4.64 \\
\hline 100 & ARL & 4.20 & 3.83 & 3.50 & 3.28 & 3.27 & 3.01 & 2.76 & 2.60 \\
\hline 1.00 & SDRL & 1.89 & 1.72 & 1.57 & 1.48 & 1.54 & 1.37 & 1.22 & 1.13 \\
\hline & ARL & 2.98 & 2.71 & 2.46 & 2.30 & 2.40 & 2.22 & 2.06 & 1.95 \\
\hline 1.20 & SDRL & 1.35 & 1.24 & 1.14 & 1.08 & 1.02 & 0.92 & 0.83 & 0.78 \\
\hline & ARL & 2.23 & 2.01 & 1.83 & 1.70 & 1.90 & 1.76 & 1.64 & 1.56 \\
\hline 1.50 & SDRL & 1.05 & 0.96 & 0.87 & 0.80 & 0.76 & 0.69 & 0.63 & 0.60 \\
\hline 2.25 & ARL & 1.20 & 1.13 & 1.08 & 1.05 & 1.19 & 1.13 & 1.08 & 1.05 \\
\hline 2.20 & SDRL & 0.44 & 0.36 & 0.28 & 0.23 & 0.40 & 0.33 & 0.27 & 0.22 \\
\hline 2.50 & ARL & 1.09 & 1.05 & 1.03 & 1.01 & 1.09 & 1.05 & 1.03 & 1.01 \\
\hline 2.50 & SDRL & 0.30 & 0.22 & 0.16 & 0.12 & 0.28 & 0.22 & 0.16 & 0.12 \\
\hline 2.75 & ARL & 1.04 & 1.02 & 1.01 & 1.00 & 1.03 & 1.02 & 1.01 & 1.00 \\
\hline 2.15 & SDRL & 0.19 & 0.12 & 0.08 & 0.06 & 0.18 & 0.13 & 0.08 & 0.06 \\
\hline 3.00 & ARL & 1.01 & 1.00 & 1.00 & 1.00 & 1.01 & 1.00 & 1.00 & 1.00 \\
\hline 3.00 & SDRL & 0.10 & 0.07 & 0.04 & 0.02 & 0.11 & 0.06 & 0.04 & 0.02 \\
\hline 3.25 & ARL & 1.00 & 1.00 & 1.00 & 1.00 & 1.00 & 1.00 & 1.00 & 1.00 \\
\hline 3.20 & SDRL & 0.06 & 0.03 & 0.01 & 0.01 & 0.05 & 0.03 & 0.02 & 0.01 \\
\hline & ARL & 1.00 & 1.00 & 1.00 & 1.00 & 1.00 & 1.00 & 1.00 & 1.00 \\
\hline 3.50 & SDRL & 0.03 & 0.01 & 0.01 & 0.01 & 0.02 & 0.01 & 0.01 & 0.00 \\
\hline 375 & ARL & 1.00 & 1.00 & 1.00 & 1.00 & 1.00 & 1.00 & 1.00 & 1.00 \\
\hline 0.25 & ARL & 61.29 & 52.22 & 45.27 & 40.63 & 103.34 & 89.54 & 76.76 & 69.54 \\
\hline 0.25 & SDRL & 69.10 & 59.21 & 51.49 & 45.93 & 134.54 & 117.61 & 102.91 & 94.93 \\
\hline 0.00 & ARL & 500.07 & 500.87 & 501.93 & 502.28 & 499.56 & 499.95 & 500.56 & 501.07 \\
\hline 0.00 & SDRL & 535.91 & 537.36 & 535.19 & 534.75 & 579.34 & 580.42 & 584.53 & 583.79 \\
\hline 0.25 & ARL & 61.29 & 52.22 & 45.27 & 40.63 & 103.34 & 89.54 & 76.76 & 69.54 \\
\hline 0.25 & SDRL & 69.10 & 59.21 & 51.49 & 45.93 & 134.54 & 117.61 & 102.91 & 94.93 \\
\hline 0.50 & ARL & 9.50 & 8.22 & 7.08 & 6.38 & 13.13 & 10.53 & 8.48 & 7.42 \\
\hline 0.50 & SDRL & 9.74 & 8.15 & 6.77 & 5.94 & 20.25 & 15.89 & 12.67 & 10.89 \\
\hline 075 & ARL & 3.83 & 3.42 & 3.06 & 2.84 & 3.50 & 3.02 & 2.66 & 2.41 \\
\hline 0.15 & SDRL & 2.91 & 2.47 & 2.11 & 1.87 & 3.97 & 3.19 & 2.52 & 2.12 \\
\hline 1.00 & ARL & 2.34 & 2.14 & 1.98 & 1.86 & 1.93 & 1.77 & 1.63 & 1.54 \\
\hline 1.00 & SDRL & 1.38 & 1.18 & 1.06 & 0.95 & 1.40 & 1.17 & 0.99 & 0.88 \\
\hline 1.25 & ARL & 1.73 & 1.60 & 1.50 & 1.42 & 1.43 & 1.34 & 1.27 & 1.22 \\
\hline 1.25 & SDRL & 0.85 & 0.75 & 0.67 & 0.61 & 0.74 & 0.64 & 0.55 & 0.49 \\
\hline & ARL & 1.40 & 1.32 & 1.24 & 1.20 & 1.20 & 1.15 & 1.10 & 1.08 \\
\hline 1.50 & SDRL & 0.59 & 0.52 & 0.46 & 0.42 & 0.46 & 0.39 & 0.33 & 0.28 \\
\hline & ARL & 1.21 & 1.15 & 1.11 & 1.08 & 1.09 & 1.06 & 1.04 & 1.02 \\
\hline 1.75 & SDRL & 0.43 & 0.37 & 0.31 & 0.27 & 0.30 & 0.24 & 0.19 & 0.15 \\
\hline & ARL & 1.10 & 1.06 & 1.04 & 1.02 & 1.03 & 1.02 & 1.01 & 1.01 \\
\hline 2.00 & SDRL & 0.30 & 0.24 & 0.19 & 0.15 & 0.18 & 0.14 & 0.10 & 0.08 \\
\hline & ARL & 1.04 & 1.02 & 1.01 & 1.01 & 1.01 & 1.01 & 1.00 & 1.00 \\
\hline 2.25 & SDRL & 0.19 & 0.14 & 0.10 & 0.08 & 0.10 & 0.07 & 0.05 & 0.03 \\
\hline 2.50 & ARL & 1.01 & 1.01 & 1.00 & 1.00 & 1.00 & 1.00 & 1.00 & 1.00 \\
\hline 2.50 & SDRL & 0.11 & 0.08 & 0.05 & 0.03 & 0.05 & 0.04 & 0.02 & 0.01 \\
\hline 2.75 & ARL & 1.00 & 1.00 & 1.00 & 1.00 & 1.00 & 1.00 & 1.00 & 1.00 \\
\hline
\end{tabular}


Table 6. The run length profiles of SCUSUM-VLRSS chart with the FIR feature for the time when the in-control ARL is 500.

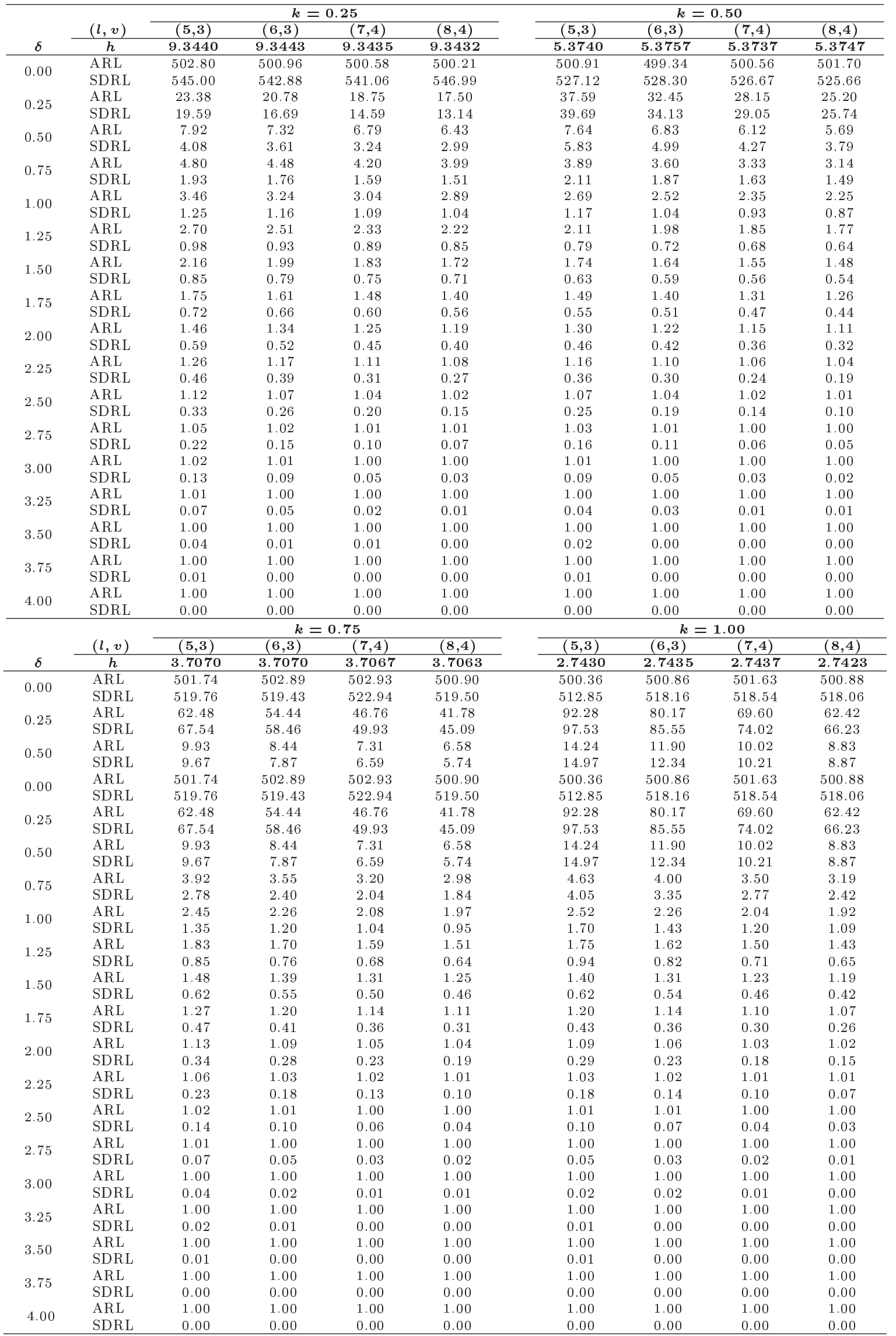


estimators, say $\overline{\bar{Y}}_{\text {VLRSS }}$ and $\hat{\operatorname{Var}}\left(\bar{Y}_{\text {VLRSS }}\right)$, respectively, defined by:

$$
\begin{aligned}
\overline{\bar{Y}}_{\mathrm{VLRSS}}=\frac{1}{q} \sum_{j=1}^{q} & \bar{Y}_{\mathrm{VLRSS}, j}, \\
\hat{\operatorname{Var}}\left(\bar{Y}_{\mathrm{VLRSS}}\right)= & \frac{1}{n m}\left(w\left(S_{Y(v: l)}^{2}+S_{Y(l-v+1: l)}^{2}\right)\right. \\
& \left.+\sum_{i=w+1}^{m-w} S_{Y(i: m)}^{2}\right)
\end{aligned}
$$

where:

$$
\begin{aligned}
\bar{Y}_{\mathrm{VLRSS}, j}= & \frac{1}{m}\left(\sum_{i=1}^{w} Y_{i(v: l) j}+\sum_{i=w+1}^{2 w} Y_{i(l-v+1: l) j}\right. \\
& \left.+\sum_{i=w+1}^{m-w} Y_{i+w(i: m) j}\right) \\
S_{Y(i: u)}^{2}= & \frac{1}{q-1} \sum_{j=1}^{q}\left(Y_{i^{\prime}(i: u) j}-\frac{1}{q} \sum_{j=1}^{q} Y_{i^{\prime}(i: u) j}\right)^{2}
\end{aligned}
$$

where $i$ and $i^{\prime}$ may or may not be equal. Under imperfect ranking, the concomitants of the study variable $Y$ corresponding to the order statistics of the auxiliary variable, $X$, are used to estimate the aforementioned parameters. From the above expressions, it is possible to obtain the unbiased estimators given that the parenthesis is replaced by the square brackets, i.e., replacing the order statistics by their corresponding concomitants. For example, replace $Y_{i(v: l) j}$ by $Y_{i[v: l] j}$ and $S_{Y(v: l)}^{2}$ by $S_{Y[v: l]}^{2}$ and, similarly, the others. For a precise estimation of the unknown parameters using the above formulae, a large number of subgroups $q$ have to be used. Once the unknown parameters get estimated, it is possible to construct the aforementioned proposed control charts for monitoring the process mean.

\section{Remarks}

The VLRSS encompasses several existing RSS schemes as it involves several parameters. On the lines of Awais and Haq [26,27], the following points may help use VLRSS to its full potential:

1. When it is possible to rank the units within sets of different sizes, it is preferable to use VLRSS with $(l>m, w=[(m-1) / 2], v=[(l+1) / 2])$ when selecting a sample of size $n$. Now, if the experimenter can rank large sets without making ranking errors, it is advisable to take $l=5,6, \cdots, 8$ or even greater, if possible. Given $m$ and $w$, as the value of $l$ increases, the value of $v$ also increases (so is the ranking cost), and it leads to a more precise mean estimator. The EWMA chart based on a more precise estimator turns out to be more sensitive. This observation is consistent with our findings in all tables. For example, given $k$, with $(m=5, w=2, l=8, v=4)$, the EWMA-VLRSS chart is the best chart;

2. When ranking costs are high and are not ignorableit is not possible to apply RSS and MRSS schemes with full confidence-yet a little ranking cost is bearable, it is preferable to use VLRSS with $(l<$ $m, w=[(m-1) / 2], v=[(l+1) / 2])$. With these choices, the VLRSS uses less identified units than those with the RSS/MRSS schemes and leads to a mean estimator, which is more precise than the mean estimator with SRS. The choice of $l$ should be as large as possible, satisfying the constraint $l<m$. For example, given $k$, with $(m=5, w=1, l=4$, $v=2$ ), the EWMA-VLRSS chart is the best chart, because, in this case, the mean estimator attains the maximum relative efficiency.

\section{Performance comparison}

In this section, the ARL and SDRL of the proposed charts are compared with those of their respective counterparts, i.e., SEWMA-VLRSS chart is compared with SEWMA-SRS, SEWMA-RSS, and SEWMAMRSS (SEWMA charts based on SRS, RSS, and MRSS), and the SCUSUM-VLRSS chart is compared with SCUSUM-SRS, SCUSUM-RSS, and SCUSUMMRSS (SCUSUM charts based on SRS, RSS, and MRSS) in terms of ARL and SDRL. The same applies here when these control charts are compared and when there are errors in ranking. For the brevity of discussion, the ARL characteristics are compared using both perfect and imperfect rankings. Note that, for the imperfect ranking case, samples are drawn from a bivariate normal distribution with different choices of $\rho$. The in-control ARLs are matched as 500 in all comparisons with $n=5$ and $r=1$.

\subsection{SEWMA and SCUSUM charts with perfect rankings}

The ARLs and SDRLs of the SEWMA-VLRSS and SCUSUM-VLRSS charts are compared with those of the SEWMA-SRS, SEWMA-RSS, SEWMA-MRSS (SEWMA based on SRS, RSS, and MRSS), SCUSUMSRS, SCUSUM-RSS, and SCUSUM-MRSS (SCUSUM based on SRS, RSS, and MRSS) charts, respectively, with different choices of $\delta, \lambda$, and $k$ in Tables 7 and 8. It is observed that, as expected, the SEWMAVLRSS (SCUSUM-VLRSS) chart performs uniformly better than the SEWMA (SCUSUM) charts based on SRS, RSS, and MRSS schemes. The sensitivity 
Table 7. The run length comparison of SEWMA-VLRSS chart with the SEWMA-SRS, SEWMA-RSS, and SEWMA-MRSS charts.

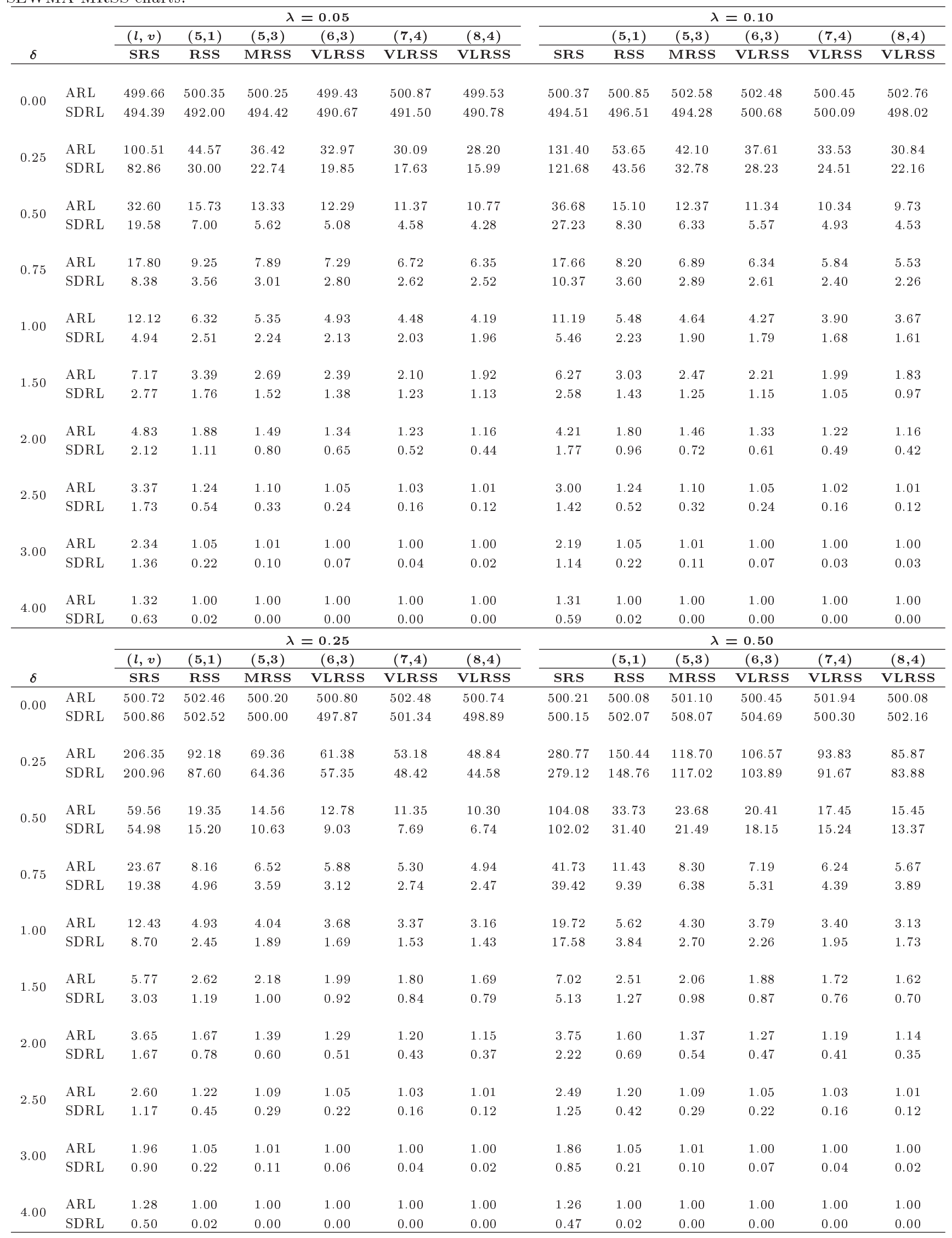


Table 8. The run length comparison of SCUSUM-VLRSS chart with the SCUSUM-SRS, SCUSUM-RSS, and SCUSUM-MRSS charts.

\begin{tabular}{|c|c|c|c|c|c|c|c|c|c|c|c|c|c|}
\hline \multirow[b]{3}{*}{$\delta$} & & \multicolumn{6}{|c|}{$k=0.25$} & \multicolumn{6}{|c|}{$k=0.50$} \\
\hline & & $(l, \boldsymbol{v})$ & $(5,1)$ & $(5,3)$ & $(6,3)$ & $(7,4)$ & $(8,4)$ & & $(5,1)$ & $(5,3)$ & $(6,3)$ & $(7,4)$ & $(8,4)$ \\
\hline & & SRS & RSS & MRSS & VLRSS & VLRSS & VLRSS & SRS & RSS & MRSS & VLRSS & VLRSS & VLRSS \\
\hline \multirow{2}{*}{0.00} & ARL & 500.86 & 500.73 & 500.80 & 500.62 & 500.72 & 500.26 & 500.43 & 501.50 & 502.65 & 500.08 & 501.11 & 499.16 \\
\hline & SDRL & 490.40 & 488.51 & 486.65 & 490.59 & 492.92 & 487.82 & 500.13 & 492.83 & 497.32 & 493.26 & 494.90 & 491.04 \\
\hline \multirow{2}{*}{0.25} & ARL & 99.60 & 43.60 & 36.00 & 32.87 & 30.02 & 28.32 & 154.81 & 60.85 & 47.16 & 41.55 & 36.91 & 33.68 \\
\hline & SDRL & 81.21 & 28.10 & 21.62 & 18.97 & 16.75 & 15.31 & 147.97 & 53.31 & 39.74 & 34.25 & 29.62 & 26.48 \\
\hline \multirow{2}{*}{0.50} & ARL & 32.44 & 15.93 & 13.64 & 12.66 & 11.76 & 11.17 & 40.86 & 14.89 & 12.17 & 11.00 & 9.96 & 9.32 \\
\hline & SDRL & 18.57 & 6.63 & 5.36 & 4.82 & 4.36 & 4.09 & 33.67 & 8.92 & 6.73 & 5.83 & 5.06 & 4.60 \\
\hline \multirow{2}{*}{0.75} & ARL & 18.17 & 9.55 & 8.25 & 7.65 & 7.11 & 6.73 & 17.99 & 7.74 & 6.48 & 5.95 & 5.50 & 5.17 \\
\hline & SDRL & 8.01 & 3.36 & 2.88 & 2.66 & 2.49 & 2.38 & 11.83 & 3.50 & 2.76 & 2.44 & 2.22 & 2.06 \\
\hline \multirow{2}{*}{1.00} & ARL & 12.49 & 6.69 & 5.71 & 5.27 & 4.85 & 4.54 & 10.81 & 5.13 & 4.37 & 4.05 & 3.73 & 3.52 \\
\hline & SDRL & 4.77 & 2.35 & 2.11 & 2.02 & 1.94 & 1.88 & 5.71 & 2.03 & 1.71 & 1.56 & 1.44 & 1.37 \\
\hline \multirow{2}{*}{1.50} & ARL & 7.56 & 3.73 & 3.02 & 2.69 & 2.37 & 2.16 & 5.89 & 2.95 & 2.48 & 2.26 & 2.05 & 1.91 \\
\hline & SDRL & 2.61 & 1.73 & 1.56 & 1.45 & 1.33 & 1.24 & 2.42 & 1.21 & 1.08 & 1.01 & 0.95 & 0.90 \\
\hline \multirow{2}{*}{2.00} & ARL & 5.21 & 2.10 & 1.65 & 1.46 & 1.31 & 1.23 & 3.98 & 1.89 & 1.54 & 1.40 & 1.28 & 1.21 \\
\hline & SDRL & 2.00 & 1.21 & 0.92 & 0.77 & 0.62 & 0.51 & 1.53 & 0.89 & 0.73 & 0.64 & 0.53 & 0.46 \\
\hline \multirow{2}{*}{2.50} & ARL & 3.70 & 1.34 & 1.14 & 1.08 & 1.04 & 1.02 & 2.93 & 1.30 & 1.13 & 1.08 & 1.04 & 1.02 \\
\hline & SDRL & 1.73 & 0.65 & 0.39 & 0.29 & 0.20 & 0.16 & 1.20 & 0.56 & 0.36 & 0.28 & 0.20 & 0.15 \\
\hline \multirow{2}{*}{3.00} & ARL & 2.62 & 1.07 & 1.02 & 1.01 & 1.00 & 1.00 & 2.23 & 1.07 & 1.02 & 1.01 & 1.00 & 1.00 \\
\hline & SDRL & 1.43 & 0.28 & 0.13 & 0.09 & 0.04 & 0.04 & 1.01 & 0.26 & 0.13 & 0.09 & 0.05 & 0.04 \\
\hline \multirow{2}{*}{4.00} & ARL & 1.43 & 1.00 & 1.00 & 1.00 & 1.00 & 1.00 & 1.38 & 1.00 & 1.00 & 1.00 & 1.00 & 1.00 \\
\hline & SDRL & 0.74 & 0.03 & 0.00 & 0.00 & 0.00 & 0.00 & 0.62 & 0.03 & 0.00 & 0.01 & 0.00 & 0.00 \\
\hline \multirow[b]{3}{*}{$\delta$} & & \multicolumn{6}{|c|}{$k=0.75$} & \multicolumn{6}{|c|}{$k=1.00$} \\
\hline & & $(l, \boldsymbol{v})$ & $(5,1)$ & $(5,3)$ & $(6,3)$ & $(7,4)$ & $(8,4)$ & & $(5,1)$ & $(5,3)$ & $(6,3)$ & $(7,4)$ & $(8,4)$ \\
\hline & & SRS & RSS & MRSS & VLRSS & VLRSS & VLRSS & SRS & RSS & MRSS & VLRSS & VLRSS & VLRSS \\
\hline \multirow{2}{*}{0.00} & ARL & 501.84 & 501.24 & 502.85 & 502.68 & 502.63 & 500.05 & 499.85 & 500.15 & 500.36 & 499.33 & 501.55 & 500.13 \\
\hline & SDRL & 498.78 & 500.01 & 499.40 & 497.36 & 501.27 & 495.78 & 500.34 & 498.37 & 497.91 & 496.82 & 500.44 & 498.09 \\
\hline \multirow{2}{*}{0.25} & ARL & 210.92 & 91.33 & 70.37 & 61.78 & 53.89 & 48.85 & 255.35 & 124.39 & 96.77 & 86.37 & 75.50 & 68.85 \\
\hline & SDRL & 207.75 & 87.58 & 66.21 & 57.04 & 49.40 & 44.55 & 252.81 & 123.01 & 94.02 & 84.06 & 72.87 & 66.29 \\
\hline \multirow{2}{*}{0.50} & ARL & 60.35 & 18.18 & 13.86 & 12.16 & 10.70 & 9.81 & 83.74 & 24.45 & 17.85 & 15.30 & 13.16 & 11.72 \\
\hline & SDRL & 56.15 & 14.05 & 10.04 & 8.45 & 7.10 & 6.29 & 81.36 & 21.59 & 15.22 & 12.52 & 10.48 & 9.08 \\
\hline 075 & ARL & 22.98 & 7.73 & 6.23 & 5.60 & 5.11 & 4.73 & 31.82 & 8.78 & 6.69 & 5.91 & 5.26 & 4.81 \\
\hline 0.10 & SDRL & 18.98 & 4.44 & 3.32 & 2.85 & 2.51 & 2.25 & 29.24 & 6.25 & 4.38 & 3.68 & 3.13 & 2.76 \\
\hline 100 & ARL & 11.94 & 4.71 & 3.92 & 3.59 & 3.28 & 3.09 & 15.02 & 4.78 & 3.84 & 3.48 & 3.16 & 2.96 \\
\hline & SDRL & 8.30 & 2.22 & 1.75 & 1.55 & 1.38 & 1.30 & 12.34 & 2.73 & 1.99 & 1.71 & 1.49 & 1.36 \\
\hline 150 & ARL & 5.55 & 2.59 & 2.19 & 2.01 & 1.84 & 1.74 & 5.80 & 2.46 & 2.08 & 1.92 & 1.77 & 1.67 \\
\hline & SDRL & 2.83 & 1.06 & 0.90 & 0.84 & 0.77 & 0.73 & 3.55 & 1.05 & 0.84 & 0.76 & 0.69 & 0.65 \\
\hline 200 & ARL & 3.55 & 1.71 & 1.45 & 1.34 & 1.25 & 1.19 & 3.42 & 1.66 & 1.44 & 1.33 & 1.24 & 1.19 \\
\hline & SDRL & 1.53 & 0.72 & 0.58 & 0.52 & 0.46 & 0.40 & 1.68 & 0.65 & 0.55 & 0.49 & 0.44 & 0.40 \\
\hline 250 & ARL & 2.57 & 1.27 & 1.12 & 1.07 & 1.04 & 1.02 & 2.43 & 1.26 & 1.12 & 1.07 & 1.04 & 1.02 \\
\hline 2.00 & SDRL & 1.07 & 0.47 & 0.33 & 0.26 & 0.20 & 0.15 & 1.04 & 0.45 & 0.33 & 0.26 & 0.20 & 0.15 \\
\hline 300 & ARL & 1.99 & 1.07 & 1.02 & 1.01 & 1.00 & 1.00 & 1.89 & 1.07 & 1.02 & 1.01 & 1.00 & 1.00 \\
\hline & SDRL & 0.82 & 0.25 & 0.13 & 0.08 & 0.05 & 0.03 & 0.75 & 0.25 & 0.13 & 0.09 & 0.04 & 0.03 \\
\hline م م & ARL & 1.33 & 1.00 & 1.00 & 1.00 & 1.00 & 1.00 & 1.32 & 1.00 & 1.00 & 1.00 & 1.00 & 1.00 \\
\hline 4.00 & SDRL & 0.51 & 0.03 & 0.00 & 0.00 & 0.00 & 0.00 & 0.48 & 0.03 & 0.01 & 0.00 & 0.00 & 0.00 \\
\hline
\end{tabular}


of the SEWMA-VLRSS/SCUSUM-VLRSS chart increases as the value of pair $(l, v)$ increases, and vice versa.

\subsection{SEWMA and SCUSUM charts with imperfect rankings}

On similar lines, the SEWMA and SCUSUM charts are compared with those based on SRS and imperfect RSS schemes. The run length profiles of the considered SEWMA and SCUSUM charts are reported in Tables 9 and 10, respectively. This time, for brevity of discussion, we consider $\lambda=0.25$ and $k=0.50$ for the SEWMA and SCUSUM charts, respectively. It is observed that, despite the presence of ranking errors, the SEWMA-VLRSS (SCUSUM-VLRSS) chart turns out to be more sensitive than its existing counterparts. As expected, the sensitivity of a control chart increases as the values of $\rho$ increase, and vice versa.

\subsection{SEWMA and SCUSUM charts with SRS}

In a situation where ranking costs are high, it may not be possible to select a sample using RSS and
MRSS schemes. However, it is possible to select a sample using VLRSS scheme with fewer identified units than those of the RSS and MRSS schemes. Hence, SEWMA-VLRSS and SCUSUM-VLRSS charts are compared with the SEWMA-SRS and SCUSUMSRS charts, respectively, for those choices of $(l, v)$ for which the ranking cost is less than that using RSS and MRSS schemes, i.e., we consider those choices of VLRSS scheme with $n=5$ for which the number of identified units is less than 25. Both perfect and imperfect rankings are used to construct SEWMA and SCUSUM charts. Under perfect rankings, the ARL and SDRL profiles of the SEWMA-SRS and SEWMA-VLRSS charts are given in Table 11, and Tables 11 and 12, it is observed that the SEWMA- and SCUSUM-VLRSS charts are better than SEWMA- and SCUSUM-SRS charts. Similarly, the run length profiles of the proposed (VLRSS) and existing (SRS) SEWMA and SCUSUM charts with the imperfect ranking are reported in Tables 13 and 14, respectively. It turns out that the proposed charts outperform their counterparts based on SRS. As expected, with an increase in the

Table 9. The run length comparison of SEWMA-VLRSS chart with SEWMA-SRS, SEWMA-RSS, and SEWMA-MRSS charts under imperfect ranking for $\lambda=0.25$.

\begin{tabular}{|c|c|c|c|c|c|c|c|c|c|c|c|c|c|c|}
\hline \multirow[b]{5}{*}{$\delta$} & \multirow[b]{5}{*}{$L$} & \multirow[b]{3}{*}{$(l, v)$} & \multicolumn{3}{|c|}{$\rho=0.25$} & \multicolumn{3}{|c|}{$\rho=0.50$} & \multicolumn{3}{|c|}{$\rho=0.75$} & \multicolumn{3}{|c|}{$\rho=0.90$} \\
\hline & & & \multirow{2}{*}{$\begin{array}{c}w=0 \\
(5,1)\end{array}$} & \multicolumn{2}{|c|}{$w=2$} & \multirow{2}{*}{$\begin{array}{c}w=0 \\
(5, \mathbf{1})\end{array}$} & \multicolumn{2}{|c|}{$w=2$} & \multirow{2}{*}{$\begin{array}{c}w=0 \\
(5,1)\end{array}$} & \multicolumn{2}{|c|}{$w=2$} & \multirow{2}{*}{$\begin{array}{c}w=0 \\
(5,1)\end{array}$} & \multicolumn{2}{|c|}{$w=2$} \\
\hline & & & & $(5,3)$ & $(7,4)$ & & $(5,3)$ & $(7,4)$ & & $(5,3)$ & $(7,4)$ & & $(5,3)$ & $(7,4)$ \\
\hline & & SRS & RSS & MRSS & VLRSS & RSS & MRSS & VLRSS & RSS & MRSS & VLRSS & RSS & MRSS & VLRSS \\
\hline & & 3.1560 & 3.1508 & 3.1515 & 3.1518 & 3.1508 & 3.1517 & 3.1524 & 3.1518 & 3.1519 & 3.1522 & 3.1572 & 3.1523 & 3.1523 \\
\hline \multirow{2}{*}{0.00} & ARL & 500.72 & 501.58 & 499.93 & 499.64 & 500.07 & 499.47 & 502.31 & 500.49 & 499.89 & 500.76 & 499.44 & 500.21 & 500.96 \\
\hline & SDRL & 500.86 & 498.78 & 496.30 & 500.16 & 501.82 & 493.61 & 499.13 & 498.04 & 496.68 & 499.31 & 495.83 & 500.79 & 495.48 \\
\hline \multirow{2}{*}{0.25} & ARL & 206.35 & 200.30 & 200.03 & 199.38 & 180.19 & 180.16 & 176.13 & 147.35 & 139.91 & 132.65 & 115.52 & 102.44 & 90.41 \\
\hline & SDRL & 200.96 & 197.01 & 194.92 & 195.98 & 176.22 & 175.74 & 172.66 & 143.83 & 136.35 & 127.58 & 110.53 & 98.66 & 85.64 \\
\hline \multirow[t]{2}{*}{0.50} & ARL & 59.56 & 56.90 & 56.63 & 56.12 & 48.61 & 47.74 & 46.07 & 35.61 & 33.16 & 31.06 & 25.95 & 22.02 & 19.29 \\
\hline & SDRL & 54.98 & 52.50 & 51.94 & 51.41 & 44.01 & 43.35 & 41.59 & 31.23 & 28.70 & 26.74 & 21.74 & 17.75 & 15.13 \\
\hline 0.75 & ARL & 23.67 & 22.45 & 22.32 & 22.26 & 19.43 & 18.69 & 18.49 & 14.38 & 13.38 & 12.47 & 10.70 & 9.33 & 8.27 \\
\hline \multirow[t]{2}{*}{1.00} & ARL & 12.43 & 11.91 & 11.83 & 11.64 & 10.35 & 10.22 & 9.96 & 7.99 & 7.54 & 7.12 & 6.21 & 5.53 & 4.97 \\
\hline & SDRL & 8.70 & 8.19 & 8.18 & 8.19 & 6.82 & 6.69 & 6.47 & 4.80 & 4.43 & 4.09 & 3.36 & 2.88 & 2.46 \\
\hline \multirow{2}{*}{1.50} & ARL & 5.77 & 5.55 & 5.54 & 5.54 & 4.98 & 4.90 & 4.80 & 4.01 & 3.81 & 3.64 & 3.24 & 2.92 & 2.66 \\
\hline & SDRL & 3.03 & 2.90 & 2.88 & 2.88 & 2.49 & 2.43 & 2.36 & 1.87 & 1.76 & 1.67 & 1.46 & 1.31 & 1.20 \\
\hline \multirow{2}{*}{2.00} & ARL & 3.65 & 3.53 & 3.51 & 3.50 & 3.19 & 3.14 & 3.09 & 2.59 & 2.46 & 2.35 & 2.10 & 1.87 & 1.71 \\
\hline & SDRL & 1.67 & 1.61 & 1.59 & 1.60 & 1.43 & 1.41 & 1.38 & 1.16 & 1.12 & 1.07 & 0.97 & 0.87 & 0.79 \\
\hline \multirow[t]{2}{*}{2.50} & ARL & 2.60 & 2.51 & 2.51 & 2.50 & 2.27 & 2.23 & 2.20 & 1.84 & 1.74 & 1.66 & 1.47 & 1.34 & 1.24 \\
\hline & SDRL & 1.17 & 1.14 & 1.13 & 1.13 & 1.04 & 1.02 & 1.01 & 0.86 & 0.81 & 0.76 & 0.66 & 0.56 & 0.47 \\
\hline \multirow{2}{*}{3.00} & ARL & 1.96 & 1.90 & 1.88 & 1.88 & 1.71 & 1.68 & 1.65 & 1.39 & 1.32 & 1.28 & 1.17 & 1.10 & 1.06 \\
\hline & SDRL & 0.90 & 0.88 & 0.88 & 0.87 & 0.79 & 0.78 & 0.76 & 0.60 & 0.55 & 0.51 & 0.40 & 0.30 & 0.23 \\
\hline
\end{tabular}


Table 10. The run length comparison of SCUSUM-VLRSS chart with SCUSUM-SRS, SCUSUM-RSS, and SCUSUM-MRSS charts under imperfect ranking for $k=0.50$.

\begin{tabular}{|c|c|c|c|c|c|c|c|c|c|c|c|c|c|c|}
\hline \multirow[b]{5}{*}{$\delta$} & \multirow[b]{5}{*}{$h$} & \multirow[b]{3}{*}{$(l, v)$} & \multicolumn{3}{|c|}{$\rho=0.25$} & \multicolumn{3}{|c|}{$\rho=0.50$} & \multicolumn{3}{|c|}{$\rho=0.75$} & \multicolumn{3}{|c|}{$\rho=0.90$} \\
\hline & & & \multirow{2}{*}{$\begin{array}{c}w=0 \\
(5,1)\end{array}$} & \multicolumn{2}{|c|}{$w=2$} & \multirow{2}{*}{$\begin{array}{c}w=0 \\
(5,1)\end{array}$} & \multicolumn{2}{|c|}{$w=2$} & \multirow{2}{*}{$\begin{array}{c}w=0 \\
(5,1)\end{array}$} & \multicolumn{2}{|c|}{$w=2$} & \multirow{2}{*}{$\begin{array}{c}w=0 \\
(5,1)\end{array}$} & \multicolumn{2}{|c|}{$w=2$} \\
\hline & & & & $(5,3)$ & $(7,4)$ & & $(5,3)$ & $(7,4)$ & & $(5,3)$ & $(7,4)$ & & $(5,3)$ & $(7,4)$ \\
\hline & & SRS & RSS & MRSS & VLRSS & RSS & MRSS & VLRSS & RSS & MRSS & VLRSS & RSS & MRSS & VLRSS \\
\hline & & 5.2910 & 5.2915 & 5.2925 & 5.2935 & 5.2927 & 5.2925 & 5.2937 & 5.2919 & 5.2924 & 5.2931 & 5.2918 & 5.2914 & 5.2926 \\
\hline \multirow{2}{*}{0.00} & ARL & 500.43 & 501.99 & 502.68 & 501.69 & 500.63 & 501.39 & 501.80 & 501.31 & 501.93 & 500.09 & 500.95 & 501.66 & 501.16 \\
\hline & SDRL & 500.13 & 502.84 & 494.43 & 500.18 & 499.63 & 501.20 & 497.50 & 497.70 & 500.64 & 497.11 & 495.87 & 494.82 & 495.17 \\
\hline \multirow{2}{*}{0.25} & ARL & 154.81 & 150.10 & 150.00 & 149.46 & 134.96 & 131.88 & 129.58 & 105.57 & 99.76 & 94.26 & 80.67 & 70.77 & 62.33 \\
\hline & SDRL & 147.97 & 143.65 & 142.35 & 143.08 & 127.39 & 124.68 & 122.40 & 98.02 & 92.67 & 86.62 & 73.03 & 63.15 & 54.64 \\
\hline \multirow{2}{*}{0.50} & ARL & 40.86 & 39.40 & 38.89 & 38.63 & 34.10 & 33.48 & 32.64 & 25.67 & 24.03 & 22.59 & 19.34 & 17.18 & 15.23 \\
\hline & SDRL & 33.67 & 31.94 & 31.54 & 31.34 & 26.78 & 26.29 & 25.43 & 18.80 & 17.36 & 15.89 & 12.87 & 10.96 & 9.26 \\
\hline \multirow{2}{*}{0.75} & ARL & 17.99 & 17.33 & 17.17 & 17.10 & 15.27 & 14.94 & 14.80 & 12.02 & 11.41 & 10.86 & 9.60 & 8.64 & 7.86 \\
\hline & SDRL & 11.83 & 11.15 & 10.84 & 10.94 & 9.31 & 9.04 & 8.90 & 6.61 & 6.18 & 5.73 & 4.83 & 4.15 & 3.61 \\
\hline \multirow{2}{*}{1.00} & ARL & 10.81 & 10.48 & 10.41 & 10.31 & 9.40 & 9.23 & 9.15 & 7.70 & 7.31 & 6.97 & 6.22 & 5.70 & 5.21 \\
\hline & SDRL & 5.71 & 5.46 & 5.41 & 5.43 & 4.67 & 4.55 & 4.45 & 3.51 & 3.27 & 3.06 & 2.62 & 2.31 & 2.08 \\
\hline \multirow{2}{*}{1.50} & ARL & 5.89 & 5.73 & 5.73 & 5.70 & 5.22 & 5.16 & 5.10 & 4.36 & 4.16 & 4.01 & 3.59 & 3.29 & 3.01 \\
\hline & SDRL & 2.42 & 2.34 & 2.34 & 2.32 & 2.08 & 2.05 & 2.01 & 1.68 & 1.61 & 1.54 & 1.40 & 1.31 & 1.22 \\
\hline \multirow[t]{2}{*}{2.00} & ARL & 3.98 & 3.89 & 3.88 & 3.86 & 3.55 & 3.49 & 3.45 & 2.94 & 2.81 & 2.68 & 2.38 & 2.14 & 1.93 \\
\hline & SDRL & 1.53 & 1.50 & 1.48 & 1.50 & 1.38 & 1.36 & 1.36 & 1.20 & 1.16 & 1.13 & 1.05 & 0.98 & 0.91 \\
\hline \multirow{2}{*}{2.50} & ARL & 2.93 & 2.85 & 2.85 & 2.83 & 2.59 & 2.54 & 2.51 & 2.09 & 1.97 & 1.88 & 1.64 & 1.47 & 1.33 \\
\hline & SDRL & 1.20 & 1.17 & 1.17 & 1.17 & 1.10 & 1.08 & 1.08 & 0.96 & 0.92 & 0.89 & 0.78 & 0.69 & 0.58 \\
\hline \multirow{2}{*}{3.00} & ARL & 2.23 & 2.17 & 2.15 & 2.14 & 1.93 & 1.91 & 1.87 & 1.54 & 1.45 & 1.39 & 1.24 & 1.14 & 1.08 \\
\hline & SDRL & 1.01 & 0.99 & 0.98 & 0.98 & 0.91 & 0.90 & 0.89 & 0.72 & 0.67 & 0.63 & 0.49 & 0.38 & 0.29 \\
\hline \multirow{2}{*}{4.00} & ARL & 1.38 & 1.34 & 1.34 & 1.33 & 1.22 & 1.20 & 1.19 & 1.07 & 1.05 & 1.04 & 1.01 & 1.00 & 1.00 \\
\hline & SDRL & 0.62 & 0.59 & 0.58 & 0.58 & 0.47 & 0.45 & 0.44 & 0.26 & 0.22 & 0.19 & 0.11 & 0.06 & 0.03 \\
\hline
\end{tabular}

value of $\rho$, the sensitivity of the SEWMA and SCUSUM charts increases, and vice versa.

\section{Illustrative example}

In this section, an illustrative example is presented to explain the working of the proposed and existing SEWMA and SCUSUM charts based on MRSS and VLRSS schemes.

A hard-bake process in conjunction with the photolithography is used in a semiconductor manufacturing process. Our objective is to establish statistical control of the flow width of the resist for this process using the proposed control charts. For this purpose, forty-five samples, each of five-wafer size (measured in microns), are drawn from an in-control process. The complete dataset is given in Montgomery [44]. Since the samples are selected with SRS, in order to generate samples using MRSS and VLRSS, the whole dataset is combined to generate a population that comprises 225 observations. Then, thirty samples, each of size five, are generated using MRSS $(l=m, v=3, w=2)$ and VLRSS $(l=6, v=3, w=2)$. These thirty samples are treated as phase-I samples. Here, under both MRSS and VLRSS, the samples are drawn using with replacement selection. These data are used to estimate the means and variances of the mean estimators with
MRSS and VLRSS. For SEWMA and SCUSUM charts, the in-control ARLs are set equal to 500 with $\lambda=0.25$ and $k=0.50$, respectively. The values of $\left(L, d_{1}\right)$ for SEWMA-MRSS and SEWMA-VLRSS charts are set to $(3.1570,3.31)$ and $(3.1579,3.31)$, respectively. Similarly, the values of $\left(k, d_{2}\right)$ for SCUSUM-MRSS and SCUSUM-VLRSS charts are set to $(5.2930,3.5)$ and $(5.2935,3.5)$, respectively. Then, the control limits of both SEWMA and SCUSUM charts are estimated using thirty phase-I samples. Now, the data for the phase-II monitoring are generated. For this purpose, from the same population, twenty samples, each of size five, under MRSS and VLRSS are generated. Then, $\delta=0.02$ is added in the last twenty samples' observations. The plotting-statistics of SEWMA and SCUSUM charts are computed for all fifty samples (obtained by MRSS and VLRSS) and are displayed in Figures 1-4.

From Figures 1-4, it can be seen that the process remains in the control state for the first thirty samples, while the control charts are issuing out-ofcontrol signals in the last twenty samples to indicate an upward shift in the underlying process mean. It is observed that SEWMA-MRSS and SEWMA-VLRSS charts detect an upward shift in the process mean at the 45 th and 42 nd samples, respectively, while SCUSUMMRSS and SCUSUM-VLRSS charts detect the same 
Table 11. The run length comparison of SEWMA-VLRSS chart with SEWMA-SRS chart.

\begin{tabular}{|c|c|c|c|c|c|c|c|c|c|}
\hline \multirow[b]{3}{*}{$\delta$} & & \multicolumn{4}{|c|}{$\lambda=0.05$} & \multicolumn{4}{|c|}{$\lambda=0.10$} \\
\hline & & $(l, v)$ & $(2,1)$ & $(3,2)$ & $(4,2)$ & & $(2,1)$ & $(3,2)$ & $(4,2)$ \\
\hline & & SRS & VLRSS & VLRSS & VLRSS & SRS & VLRSS & VLRSS & VLRSS \\
\hline \multirow{2}{*}{0.00} & ARL & 499.66 & 499.32 & 500.62 & 499.16 & 500.37 & 500.76 & 500.68 & 500.31 \\
\hline & SDRL & 494.39 & 486.43 & 492.10 & 491.01 & 494.51 & 496.14 & 495.93 & 494.79 \\
\hline \multirow{2}{*}{0.25} & ARL & 100.51 & 52.99 & 43.71 & 40.14 & 131.40 & 66.29 & 52.12 & 47.97 \\
\hline & SDRL & 82.86 & 37.77 & 29.17 & 25.84 & 121.68 & 55.93 & 42.36 & 38.18 \\
\hline \multirow{2}{*}{0.50} & ARL & 32.60 & 18.20 & 15.53 & 14.52 & 36.68 & 18.09 & 14.95 & 13.76 \\
\hline & SDRL & 19.58 & 8.69 & 6.87 & 6.28 & 27.23 & 10.73 & 8.28 & 7.33 \\
\hline \multirow{2}{*}{0.75} & ARL & 17.80 & 10.64 & 9.14 & 8.58 & 17.66 & 9.59 & 8.07 & 7.54 \\
\hline & SDRL & 8.38 & 4.20 & 3.50 & 3.27 & 10.37 & 4.40 & 3.53 & 3.20 \\
\hline \multirow{2}{*}{1.00} & ARL & 12.12 & 7.34 & 6.30 & 5.86 & 11.19 & 6.39 & 5.44 & 5.08 \\
\hline & SDRL & 4.94 & 2.82 & 2.48 & 2.36 & 5.46 & 2.62 & 2.23 & 2.08 \\
\hline \multirow{2}{*}{1.50} & ARL & 7.17 & 4.11 & 3.38 & 3.07 & 6.27 & 3.61 & 3.01 & 2.77 \\
\hline & SDRL & 2.77 & 1.94 & 1.75 & 1.65 & 2.58 & 1.60 & 1.42 & 1.35 \\
\hline \multirow{2}{*}{2.00} & ARL & 4.83 & 2.39 & 1.88 & 1.69 & 4.21 & 2.24 & 1.80 & 1.63 \\
\hline & SDRL & 2.12 & 1.39 & 1.10 & 0.97 & 1.77 & 1.16 & 0.95 & 0.85 \\
\hline \multirow{2}{*}{2.50} & ARL & 3.37 & 1.51 & 1.25 & 1.16 & 3.00 & 1.48 & 1.24 & 1.16 \\
\hline & SDRL & 1.73 & 0.83 & 0.54 & 0.44 & 1.42 & 0.74 & 0.52 & 0.42 \\
\hline \multirow{2}{*}{3.00} & $\mathrm{ARL}$ & 2.34 & 1.14 & 1.05 & 1.03 & 2.19 & 1.14 & 1.05 & 1.03 \\
\hline & SDRL & 1.36 & 0.40 & 0.22 & 0.17 & 1.14 & 0.39 & 0.22 & 0.16 \\
\hline \multirow{2}{*}{4.00} & ARL & 1.32 & 1.00 & 1.00 & 1.00 & 1.31 & 1.00 & 1.00 & 1.00 \\
\hline & SDRL & 0.63 & 0.06 & 0.02 & 0.01 & 0.59 & 0.06 & 0.02 & 0.01 \\
\hline \multirow[b]{3}{*}{$\delta$} & & \multicolumn{4}{|c|}{$\lambda=0.25$} & \multicolumn{4}{|c|}{$\lambda=0.50$} \\
\hline & & $(l, \boldsymbol{v})$ & $(2,1)$ & $(3,2)$ & $(4,2)$ & & $(2,1)$ & $(3,2)$ & $(4,2)$ \\
\hline & & SRS & VLRSS & VLRSS & VLRSS & SRS & VLRSS & VLRSS & VLRSS \\
\hline \multirow{2}{*}{0.00} & ARL & 500.72 & 500.97 & 501.83 & 502.89 & 500.21 & 500.45 & 500.41 & 500.25 \\
\hline & SDRL & 500.86 & 496.30 & 497.12 & 501.76 & 500.15 & 501.39 & 500.87 & 503.79 \\
\hline \multirow{2}{*}{0.25} & ARL & 206.35 & 112.27 & 89.38 & 80.32 & 280.77 & 175.36 & 145.23 & 136.20 \\
\hline & SDRL & 200.96 & 108.33 & 85.21 & 75.99 & 279.12 & 172.28 & 143.50 & 134.42 \\
\hline \multirow{2}{*}{0.50} & ARL & 59.56 & 24.45 & 18.90 & 16.81 & 104.08 & 43.67 & 32.37 & 28.74 \\
\hline & SDRL & 54.98 & 20.19 & 14.74 & 12.75 & 102.02 & 41.58 & 30.11 & 26.79 \\
\hline \multirow{2}{*}{0.75} & $\mathrm{ARL}$ & 23.67 & 10.12 & 8.05 & 7.32 & 41.73 & 15.30 & 11.19 & 9.88 \\
\hline & SDRL & 19.38 & 6.57 & 4.78 & 4.19 & 39.42 & 13.18 & 9.17 & 7.91 \\
\hline \multirow[t]{2}{*}{1.00} & $\mathrm{ARL}$ & 12.43 & 5.92 & 4.89 & 4.50 & 19.72 & 7.32 & 5.55 & 4.98 \\
\hline & SDRL & 8.70 & 3.14 & 2.44 & 2.17 & 17.58 & 5.46 & 3.81 & 3.29 \\
\hline 1.50 & ARL & 5.77 & 3.10 & 2.61 & 2.42 & 7.02 & 3.07 & 2.49 & 2.30 \\
\hline & SDRL & 3.03 & 1.40 & 1.17 & 1.10 & 5.13 & 1.67 & 1.26 & 1.13 \\
\hline 2.00 & ARL & 3.65 & 2.00 & 1.67 & 1.53 & 3.75 & 1.89 & 1.60 & 1.49 \\
\hline & SDRL & 1.67 & 0.93 & 0.77 & 0.70 & 2.22 & 0.87 & 0.69 & 0.62 \\
\hline 2.50 & $\mathrm{ARL}$ & 2.60 & 1.41 & 1.21 & 1.15 & 2.49 & 1.38 & 1.20 & 1.14 \\
\hline & SDRL & 1.17 & 0.61 & 0.45 & 0.38 & 1.25 & 0.56 & 0.42 & 0.36 \\
\hline 3.00 & ARL & 1.96 & 1.13 & 1.05 & 1.03 & 1.86 & 1.13 & 1.05 & 1.03 \\
\hline & SDRL & 0.90 & 0.36 & 0.21 & 0.16 & 0.85 & 0.34 & 0.21 & 0.16 \\
\hline 4.00 & $\mathrm{ARL}$ & 1.28 & 1.00 & 1.00 & 1.00 & 1.26 & 1.00 & 1.00 & 1.00 \\
\hline & SDRL & 0.50 & 0.07 & 0.02 & 0.01 & 0.47 & 0.07 & 0.03 & 0.01 \\
\hline
\end{tabular}


Table 12. The run length comparison of SCUSUM-VLRSS chart with SCUSUM-SRS chart.

\begin{tabular}{|c|c|c|c|c|c|c|c|c|c|}
\hline \multirow[b]{3}{*}{$\delta$} & \multirow[b]{3}{*}{$\boldsymbol{h}$} & \multicolumn{4}{|c|}{$k=0.25$} & \multicolumn{4}{|c|}{$k=0.50$} \\
\hline & & $(l, v)$ & $(2,1)$ & $(3,2)$ & $(4,2)$ & & $(2,1)$ & $(3,2)$ & $(4,2)$ \\
\hline & & SRS & VLRSS & VLRSS & VLRSS & SRS & VLRSS & VLRSS & VLRSS \\
\hline \multirow{2}{*}{0.00} & ARL & 500.86 & 500.18 & 501.73 & 500.67 & 500.43 & 500.20 & 500.71 & 500.14 \\
\hline & SDRL & 490.40 & 492.28 & 484.81 & 488.69 & 500.13 & 498.31 & 503.18 & 499.89 \\
\hline \multirow{2}{*}{0.25} & ARL & 99.60 & 52.45 & 43.30 & 39.93 & 154.81 & 76.63 & 60.27 & 54.50 \\
\hline & SDRL & 81.21 & 36.17 & 28.12 & 24.94 & 147.97 & 69.39 & 52.27 & 46.70 \\
\hline \multirow{2}{*}{0.50} & ARL & 32.44 & 18.53 & 15.86 & 14.86 & 40.86 & 18.42 & 14.86 & 13.58 \\
\hline & SDRL & 18.57 & 8.21 & 6.62 & 6.01 & 33.67 & 12.04 & 8.94 & 7.88 \\
\hline \multirow{2}{*}{0.75} & ARL & 18.17 & 10.99 & 9.55 & 8.96 & 17.99 & 9.18 & 7.68 & 7.13 \\
\hline & SDRL & 8.01 & 4.00 & 3.36 & 3.13 & 11.83 & 4.48 & 3.49 & 3.15 \\
\hline \multirow{2}{*}{1.00} & ARL & 12.49 & 7.72 & 6.67 & 6.22 & 10.81 & 6.02 & 5.11 & 4.77 \\
\hline & SDRL & 4.77 & 2.67 & 2.35 & 2.23 & 5.71 & 2.50 & 2.03 & 1.85 \\
\hline \multirow{2}{*}{1.50} & ARL & 7.56 & 4.47 & 3.71 & 3.40 & 5.89 & 3.46 & 2.94 & 2.74 \\
\hline & SDRL & 2.61 & 1.88 & 1.74 & 1.66 & 2.42 & 1.35 & 1.20 & 1.14 \\
\hline \multirow{2}{*}{2.00} & ARL & 5.21 & 2.69 & 2.11 & 1.89 & 3.98 & 2.27 & 1.88 & 1.73 \\
\hline & SDRL & 2.00 & 1.46 & 1.21 & 1.09 & 1.53 & 1.02 & 0.89 & 0.83 \\
\hline \multirow{2}{*}{2.50} & ARL & 3.70 & 1.67 & 1.34 & 1.23 & 2.93 & 1.57 & 1.30 & 1.22 \\
\hline & SDRL & 1.73 & 0.94 & 0.65 & 0.53 & 1.20 & 0.75 & 0.55 & 0.47 \\
\hline \multirow{2}{*}{3.00} & ARL & 2.62 & 1.21 & 1.07 & 1.04 & 2.23 & 1.19 & 1.07 & 1.04 \\
\hline & SDRL & 1.43 & 0.49 & 0.28 & 0.21 & 1.01 & 0.44 & 0.27 & 0.20 \\
\hline \multirow{2}{*}{4.00} & ARL & 1.43 & 1.01 & 1.00 & 1.00 & 1.38 & 1.01 & 1.00 & 1.00 \\
\hline & SDRL & 0.74 & 0.09 & 0.03 & 0.01 & 0.62 & 0.09 & 0.03 & 0.01 \\
\hline \multirow[b]{3}{*}{$\delta$} & \multirow[b]{3}{*}{$h$} & \multicolumn{4}{|c|}{$k=0.75$} & \multicolumn{4}{|c|}{$k=1.00$} \\
\hline & & $(l, v)$ & $(2,1)$ & $(3,2)$ & $(4,2)$ & & $(2,1)$ & $(3,2)$ & $(4,2)$ \\
\hline & & SRS & VLRSS & VLRSS & VLRSS & SRS & VLRSS & VLRSS & VLRSS \\
\hline \multirow{2}{*}{0.00} & ARL & 501.84 & 502.50 & 502.97 & 500.93 & 499.85 & 499.56 & 500.75 & 502.78 \\
\hline & SDRL & 498.78 & 499.33 & 500.89 & 501.22 & 500.34 & 498.18 & 497.22 & 502.07 \\
\hline \multirow{2}{*}{0.25} & ARL & 210.92 & 113.71 & 90.64 & 80.81 & 255.35 & 149.44 & 121.97 & 111.38 \\
\hline & SDRL & 207.75 & 109.91 & 87.06 & 75.61 & 252.81 & 147.02 & 118.80 & 109.08 \\
\hline \multirow{2}{*}{0.50} & ARL & 60.35 & 23.70 & 18.04 & 16.04 & 83.74 & 32.93 & 24.29 & 21.36 \\
\hline & SDRL & 56.15 & 19.66 & 13.98 & 12.11 & 81.36 & 30.11 & 21.43 & 18.65 \\
\hline 075 & ARL & 22.98 & 9.64 & 7.66 & 6.98 & 31.82 & 11.45 & 8.71 & 7.70 \\
\hline 0.15 & SDRL & 18.98 & 6.18 & 4.45 & 3.94 & 29.24 & 8.80 & 6.24 & 5.29 \\
\hline 100 & ARL & 11.94 & 5.67 & 4.69 & 4.33 & 15.02 & 5.95 & 4.75 & 4.31 \\
\hline & SDRL & 8.30 & 2.89 & 2.23 & 1.99 & 12.34 & 3.74 & 2.70 & 2.36 \\
\hline 1.50 & ARL & 5.55 & 3.04 & 2.59 & 2.40 & 5.80 & 2.89 & 2.44 & 2.27 \\
\hline & SDRL & 2.83 & 1.26 & 1.07 & 0.99 & 3.55 & 1.31 & 1.04 & 0.95 \\
\hline 2.00 & ARL & 3.55 & 2.02 & 1.72 & 1.59 & 3.42 & 1.92 & 1.66 & 1.55 \\
\hline 2.00 & SDRL & 1.53 & 0.84 & 0.71 & 0.66 & 1.68 & 0.76 & 0.65 & 0.60 \\
\hline 2.50 & ARL & 2.57 & 1.47 & 1.27 & 1.19 & 2.43 & 1.45 & 1.26 & 1.19 \\
\hline 2.50 & SDRL & 1.07 & 0.59 & 0.47 & 0.41 & 1.04 & 0.55 & 0.45 & 0.40 \\
\hline 300 & ARL & 1.99 & 1.18 & 1.07 & 1.04 & 1.89 & 1.17 & 1.07 & 1.04 \\
\hline 3.00 & SDRL & 0.82 & 0.39 & 0.25 & 0.19 & 0.75 & 0.38 & 0.25 & 0.19 \\
\hline 400 & ARL & 1.33 & 1.01 & 1.00 & 1.00 & 1.32 & 1.01 & 1.00 & 1.00 \\
\hline & SDRL & 0.51 & 0.09 & 0.03 & 0.01 & 0.48 & 0.08 & 0.03 & 0.02 \\
\hline
\end{tabular}


Table 13. The run length comparison of SEWMA-VLRSS chart with SEWMA-SRS chart under imperfect ranking for $\lambda=0.25$.

\begin{tabular}{|c|c|c|c|c|c|c|c|c|c|c|c|c|c|c|}
\hline \multirow[b]{4}{*}{$\delta$} & \multirow[b]{2}{*}{$(l, v)$} & \multirow[b]{2}{*}{$(0,0)$} & \multicolumn{3}{|c|}{$\rho=0.25$} & \multicolumn{3}{|c|}{$\rho=0.50$} & \multicolumn{3}{|c|}{$\rho=0.75$} & \multicolumn{3}{|c|}{$\rho=0.90$} \\
\hline & & & $(2,1)$ & $(3,2)$ & $(4,2)$ & $(2,1)$ & $(3,2)$ & $(4,2)$ & $(2,1)$ & $(3,2)$ & $(4,2)$ & $(2,1)$ & $(3,2)$ & $(4,2)$ \\
\hline & & SRS & VLRSS & VLRSS & VLRSS & VLRSS & VLRSS & VLRSS & VLRSS & VLRSS & VLRSS & VLRSS & VLRSS & VLRSS \\
\hline & $L$ & 3.1560 & 3.1505 & 3.1505 & 3.1508 & 3.1515 & 3.1511 & 3.1510 & 3.1514 & 3.1512 & 3.1512 & 3.1519 & 3.1519 & 3.1520 \\
\hline \multirow{2}{*}{0.00} & ARL & 500.72 & 501.16 & 499.86 & 500.46 & 500.09 & 499.78 & 499.80 & 499.73 & 500.05 & 501.67 & 499.77 & 501.17 & 500.47 \\
\hline & SDRL & 500.86 & 496.30 & 496.88 & 496.95 & 492.92 & 495.39 & 493.58 & 495.24 & 492.21 & 494.59 & 494.96 & 499.63 & 497.19 \\
\hline \multirow{2}{*}{0.25} & ARL & 206.35 & 201.12 & 199.02 & 198.13 & 185.43 & 180.99 & 179.38 & 156.63 & 148.55 & 142.21 & 131.05 & 115.14 & 109.16 \\
\hline & SDRL & 200.96 & 197.13 & 195.72 & 195.03 & 181.49 & 176.87 & 175.81 & 153.83 & 143.06 & 137.80 & 126.76 & 110.68 & 104.09 \\
\hline \multirow{2}{*}{0.50} & ARL & 59.56 & 57.06 & 56.75 & 56.59 & 50.30 & 49.06 & 48.14 & 39.36 & 35.89 & 34.39 & 30.74 & 25.76 & 24.13 \\
\hline & SDRL & 54.98 & 52.78 & 52.19 & 51.57 & 45.81 & 44.80 & 43.36 & 34.63 & 31.40 & 29.79 & 26.08 & 21.35 & 19.87 \\
\hline \multirow{2}{*}{0.75} & ARL & 23.67 & 22.54 & 22.34 & 22.27 & 20.00 & 19.22 & 19.01 & 15.66 & 14.28 & 13.79 & 12.34 & 10.63 & 9.99 \\
\hline & SDRL & 19.38 & 18.25 & 18.15 & 18.00 & 15.74 & 15.05 & 15.02 & 11.59 & 10.44 & 9.90 & 8.59 & 7.01 & 6.46 \\
\hline \multirow{2}{*}{1.00} & $\mathrm{ARL}$ & 12.43 & 12.04 & 11.95 & 11.88 & 10.73 & 10.41 & 10.32 & 8.56 & 8.01 & 7.77 & 7.05 & 6.21 & 5.88 \\
\hline & SDRL & 8.70 & 8.30 & 8.23 & 8.16 & 7.13 & 6.86 & 6.74 & 5.25 & 4.78 & 4.62 & 4.03 & 3.34 & 3.12 \\
\hline \multirow{2}{*}{1.50} & ARL & 5.77 & 5.60 & 5.57 & 5.53 & 5.10 & 4.98 & 4.92 & 4.27 & 4.01 & 3.92 & 3.61 & 3.24 & 3.10 \\
\hline & SDRL & 3.03 & 2.93 & 2.92 & 2.88 & 2.56 & 2.50 & 2.43 & 2.03 & 1.87 & 1.81 & 1.64 & 1.46 & 1.40 \\
\hline \multirow{2}{*}{2.00} & ARL & 3.65 & 3.55 & 3.53 & 3.52 & 3.26 & 3.19 & 3.17 & 2.75 & 2.60 & 2.53 & 2.34 & 2.09 & 1.99 \\
\hline & SDRL & 1.67 & 1.62 & 1.61 & 1.61 & 1.47 & 1.43 & 1.43 & 1.23 & 1.17 & 1.14 & 1.07 & 0.97 & 0.92 \\
\hline \multirow{2}{*}{2.50} & $\mathrm{ARL}$ & 2.60 & 2.53 & 2.51 & 2.50 & 2.32 & 2.27 & 2.26 & 1.95 & 1.84 & 1.78 & 1.64 & 1.48 & 1.41 \\
\hline & SDRL & 1.17 & 1.14 & 1.14 & 1.13 & 1.06 & 1.04 & 1.03 & 0.91 & 0.85 & 0.82 & 0.76 & 0.66 & 0.61 \\
\hline \multirow{2}{*}{3.00} & ARL & 1.96 & 1.90 & 1.89 & 1.89 & 1.75 & 1.70 & 1.69 & 1.47 & 1.39 & 1.36 & 1.27 & 1.16 & 1.13 \\
\hline & SDRL & 0.90 & 0.88 & 0.88 & 0.88 & 0.81 & 0.79 & 0.78 & 0.66 & 0.60 & 0.57 & 0.50 & 0.39 & 0.35 \\
\hline \multirow{2}{*}{4.00} & ARL & 1.28 & 1.24 & 1.24 & 1.24 & 1.17 & 1.15 & 1.14 & 1.07 & 1.05 & 1.04 & 1.02 & 1.01 & 1.00 \\
\hline & SDRL & 0.50 & 0.48 & 0.47 & 0.47 & 0.40 & 0.38 & 0.37 & 0.26 & 0.21 & 0.20 & 0.14 & 0.08 & 0.06 \\
\hline
\end{tabular}

Table 14. The run length comparison of SCUSUM-VLRSS chart with SCUSUM-SRS chart under imperfect ranking for $k=0.50$.

\begin{tabular}{|c|c|c|c|c|c|c|c|c|c|c|c|c|c|c|}
\hline \multirow[b]{5}{*}{$\delta$} & \multirow[b]{5}{*}{$h$} & \multirow[b]{3}{*}{$(l, v)$} & \multicolumn{3}{|c|}{$\rho=0.25$} & \multicolumn{3}{|c|}{$\rho=0.50$} & \multicolumn{3}{|c|}{$\rho=0.75$} & \multicolumn{3}{|c|}{$\rho=0.90$} \\
\hline & & & \multirow{2}{*}{$\begin{array}{c}w=0 \\
(2,1)\end{array}$} & \multicolumn{2}{|c|}{$w=2$} & \multirow{2}{*}{$\begin{array}{l}w=0 \\
(2,1)\end{array}$} & \multicolumn{2}{|c|}{$w=2$} & \multirow{2}{*}{$\begin{array}{c}w=0 \\
(2,1)\end{array}$} & \multicolumn{2}{|c|}{$w=2$} & \multirow{2}{*}{$\begin{array}{c}w=0 \\
(2,1)\end{array}$} & \multicolumn{2}{|c|}{$w=2$} \\
\hline & & & & $(3,2)$ & $(4,2)$ & & $(3,2)$ & $(4,2)$ & & $(3,2)$ & $(4,2)$ & & $(3,2)$ & $(4,2)$ \\
\hline & & SRS & VLRSS & VLRSS & VLRSS & VLRSS & VLRSS & VLRSS & VLRSS & VLRSS & VLRSS & VLRSS & VLRSS & VLRSS \\
\hline & & 5.2910 & 5.2911 & 5.2916 & 5.2918 & 5.2919 & 5.2913 & 5.2914 & 5.2915 & 5.2905 & 5.2911 & 5.2908 & 5.2914 & 5.2916 \\
\hline \multirow{2}{*}{0.00} & ARL & 500.43 & 501.66 & 501.96 & 500.13 & 501.84 & 500.72 & 501.11 & 500.76 & 501.56 & 500.89 & 500.00 & 501.15 & 499.63 \\
\hline & SDRL & 500.13 & 494.04 & 495.18 & 496.14 & 500.20 & 497.93 & 500.57 & 497.06 & 500.39 & 501.72 & 491.27 & 493.49 & 495.51 \\
\hline \multirow{2}{*}{0.25} & ARL & 154.81 & 151.13 & 149.58 & 149.27 & 136.73 & 133.23 & 132.38 & 113.21 & 106.37 & 101.97 & 92.35 & 81.37 & 76.09 \\
\hline & SDRL & 147.97 & 144.07 & 142.20 & 143.31 & 130.63 & 125.65 & 125.29 & 105.38 & 99.58 & 94.52 & 84.91 & 73.47 & 67.76 \\
\hline \multirow{2}{*}{0.50} & ARL & 40.86 & 39.52 & 39.18 & 38.73 & 34.76 & 34.14 & 33.74 & 27.71 & 25.64 & 24.80 & 22.35 & 19.57 & 18.29 \\
\hline & SDRL & 33.67 & 32.23 & 31.90 & 31.51 & 27.35 & 27.02 & 26.59 & 20.57 & 18.79 & 18.09 & 15.70 & 13.14 & 12.05 \\
\hline \multirow{2}{*}{0.75} & ARL & 17.99 & 17.35 & 17.32 & 17.30 & 15.62 & 15.27 & 15.15 & 12.88 & 12.03 & 11.75 & 10.70 & 9.60 & 9.10 \\
\hline & SDRL & 11.83 & 11.11 & 11.15 & 11.15 & 9.61 & 9.40 & 9.24 & 7.32 & 6.62 & 6.40 & 5.61 & 4.79 & 4.45 \\
\hline \multirow{2}{*}{1.00} & ARL & 10.81 & 10.52 & 10.48 & 10.44 & 9.63 & 9.42 & 9.32 & 8.11 & 7.67 & 7.47 & 6.93 & 6.24 & 6.00 \\
\hline & SDRL & 5.71 & 5.51 & 5.48 & 5.47 & 4.86 & 4.69 & 4.59 & 3.77 & 3.51 & 3.36 & 3.01 & 2.61 & 2.48 \\
\hline \multirow{2}{*}{1.50} & ARL & 5.89 & 5.76 & 5.76 & 5.72 & 5.33 & 5.25 & 5.18 & 4.59 & 4.37 & 4.25 & 3.97 & 3.59 & 3.44 \\
\hline & SDRL & 2.42 & 2.36 & 2.36 & 2.34 & 2.13 & 2.09 & 2.07 & 1.80 & 1.69 & 1.65 & 1.53 & 1.40 & 1.35 \\
\hline \multirow{2}{*}{2.00} & ARL & 3.98 & 3.90 & 3.89 & 3.86 & 3.62 & 3.55 & 3.52 & 3.10 & 2.94 & 2.87 & 2.66 & 2.38 & 2.27 \\
\hline & SDRL & 1.53 & 1.51 & 1.49 & 1.49 & 1.40 & 1.39 & 1.37 & 1.25 & 1.20 & 1.18 & 1.12 & 1.05 & 1.02 \\
\hline \multirow{2}{*}{2.50} & ARL & 2.93 & 2.86 & 2.86 & 2.85 & 2.62 & 2.58 & 2.57 & 2.24 & 2.10 & 2.04 & 1.86 & 1.64 & 1.57 \\
\hline & SDRL & 1.20 & 1.17 & 1.17 & 1.18 & 1.12 & 1.10 & 1.10 & 1.00 & 0.97 & 0.95 & 0.88 & 0.78 & 0.74 \\
\hline \multirow[t]{2}{*}{3.00} & ARL & 2.23 & 2.17 & 2.16 & 2.16 & 1.98 & 1.93 & 1.93 & 1.64 & 1.54 & 1.50 & 1.38 & 1.24 & 1.19 \\
\hline & SDRL & 1.01 & 0.98 & 0.99 & 0.98 & 0.93 & 0.91 & 0.90 & 0.79 & 0.73 & 0.70 & 0.61 & 0.49 & 0.44 \\
\hline \multirow{2}{*}{4.00} & ARL & 1.38 & 1.35 & 1.34 & 1.34 & 1.24 & 1.22 & 1.21 & 1.10 & 1.07 & 1.06 & 1.03 & 1.01 & 1.01 \\
\hline & SDRL & 0.62 & 0.59 & 0.59 & 0.59 & 0.50 & 0.47 & 0.47 & 0.32 & 0.26 & 0.24 & 0.18 & 0.11 & 0.09 \\
\hline
\end{tabular}



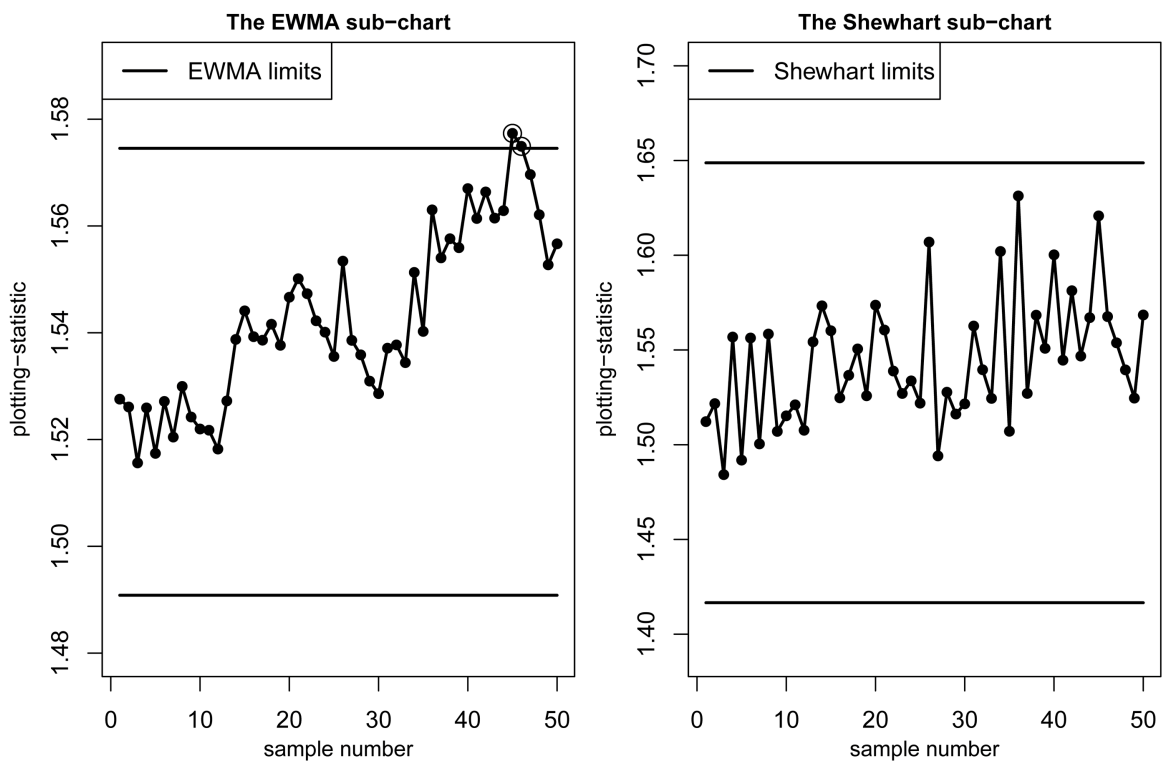

Figure 1. The SEWMA-MRSS chart for the real data.
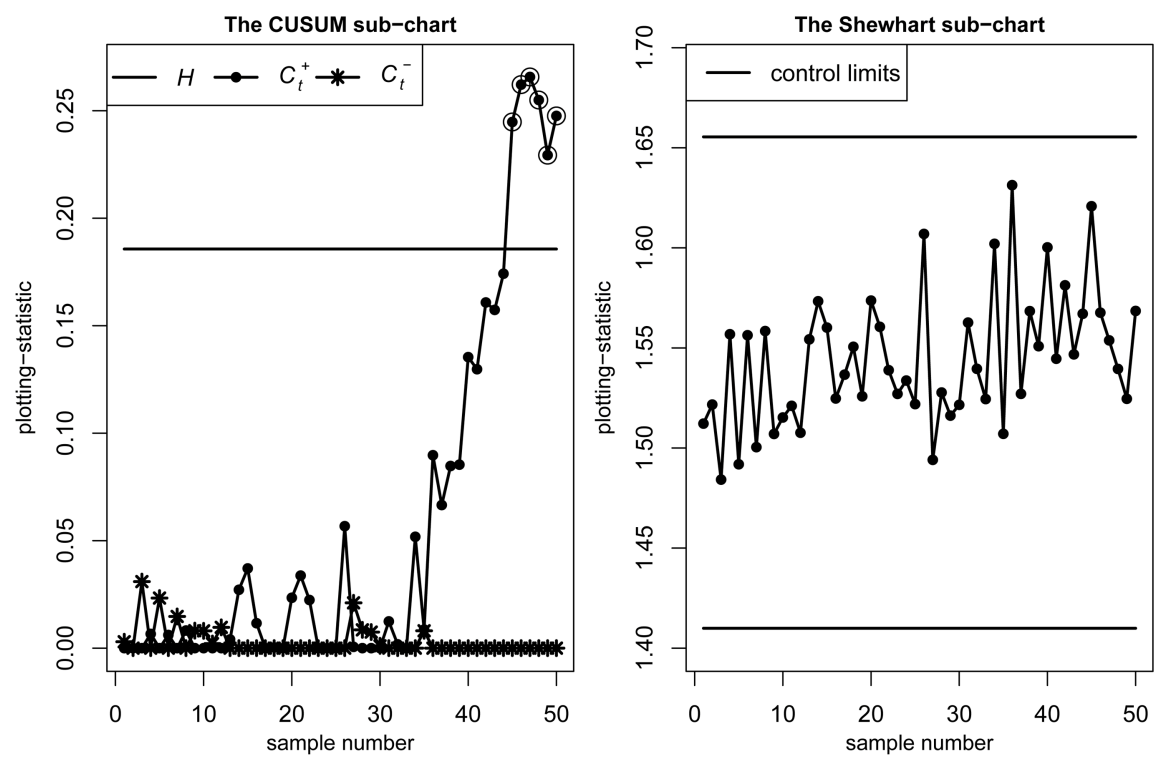

Figure 2. The SCUSUM-MRSS chart for the real data.

shift at the 45 th and 42 nd samples, respectively. Note that the median and varied $L$ ranked set samples are independently drawn; thus, their charts have different behavior. However, both schemes' samples (last twenty) are contaminated with the same value of $\delta$. This is the reason why the Shewhart subcharts with SEWMA- and SCUSUM-VLRSS charts are issuing out-of-control signals while their counterparts do not.

\section{Conclusion}

This study proposed new SEWMA and SCUSUM charts using VLRSS and IVLRSS schemes for monitor- ing the process mean. The run length characteristics of the proposed charts were computed using Monte Carlo simulations. The run length performances of the proposed charts were compared with those of their existing counterparts based on SRS, RSS, and MRSS. It was found that the proposed charts made uniform improvement over their analogous charts. Thus, it was recommended using the proposed chart, when possible, to efficiently monitor infrequent changes in the process mean of a normally distributed process.

It is possible to develop adaptive EWMA and adaptive CUSUM charts with VLRSS for the process mean when detecting changes in a range of mean shift sizes. Moreover, dual EWMA and dual CUSUM 

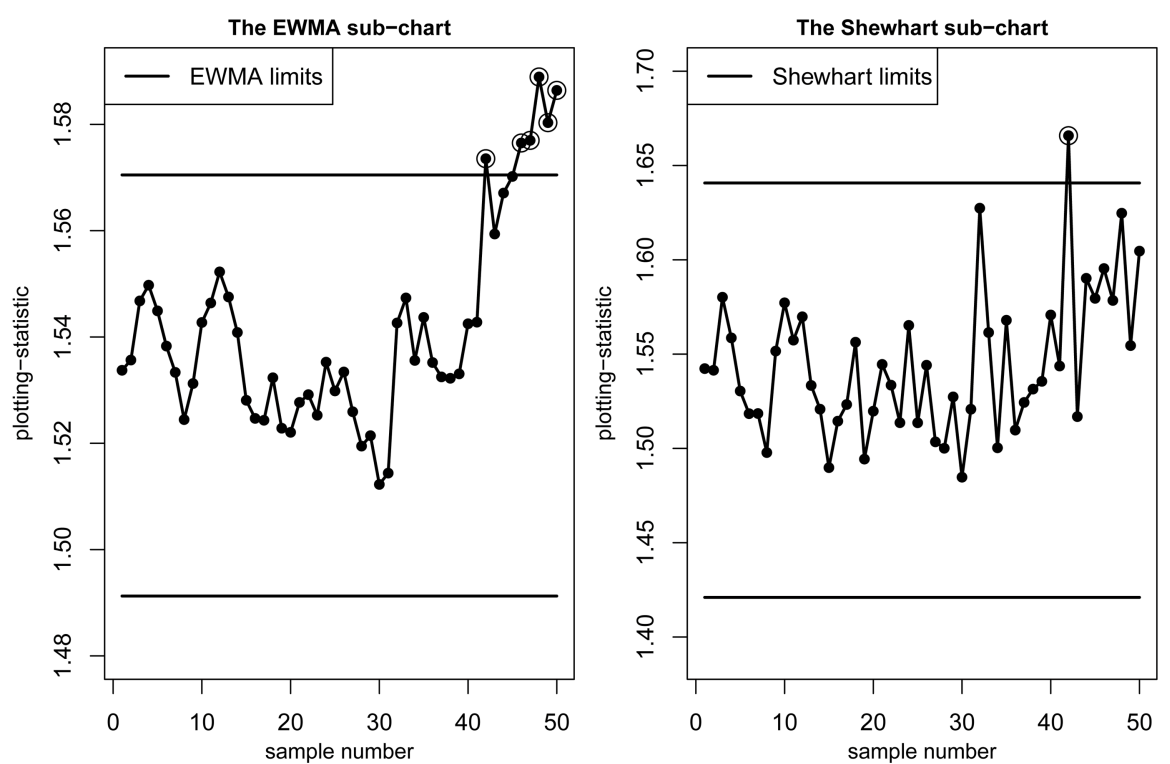

Figure 3. The SEWMA-VLRSS chart for the real data.
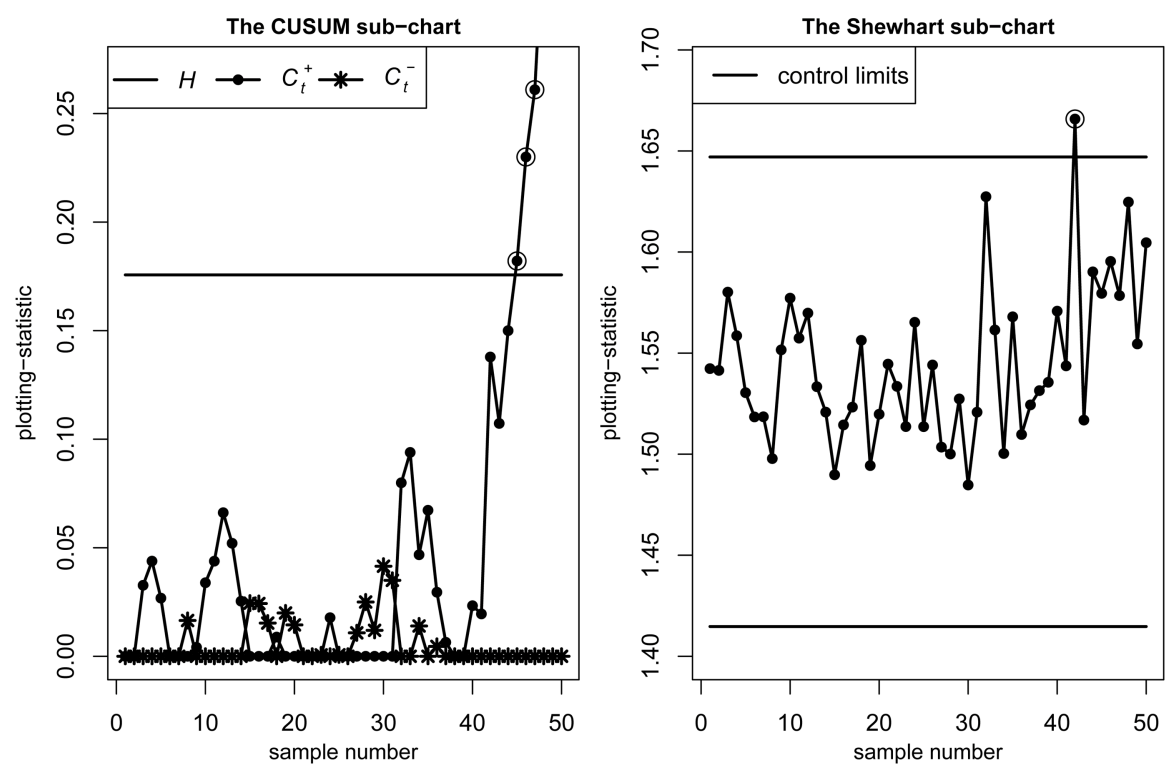

Figure 4. The SCUSUM-VLRSS chart for the real data.

charts can be constructed with VLRSS for efficiently monitoring the process mean.

\section{References}

1. Page, E.S. "Continuous inspection schemes", Biometrika, 41(1-2), pp. 100-115 (1954).

2. Lucas, J.M. and Crosier, R.B. "Fast initial response for CUSUM quality-control schemes: Give your CUSUM a head start", Technometrics, 24(3), pp. 199-205 (1982).

3. Lucas, J.M. "Combined Shewhart-CUSUM quality control schemes", Journal of Quality Technology, 14(2), pp. 51-59 (1982).

4. Roberts, W.S. "Control chart tests based on geometric moving averages", Technometrics, 1(3), pp. 239-250 (1959).

5. Lucas, J.M. and Saccucci, M.S. "Exponentially weighted moving average control schemes: Properties and enhancements", Technometrics, 32(1), pp. 1-12 (1990).

6. Knoth, S. "Fast initial response features for EWMA control charts", Statistical Papers, 46(1), pp. 47-64 (2005).

7. Chiu, W.C. "Generally weighted moving average control charts with fast initial response features", Journal of Applied Statistics, 36(3), pp. 255-275 (2009).

8. Abbas, N., Riaz, M., and Does, R.J.M.M. "Mixed exponentially weighted moving average cumulative sum 
charts for process monitoring", Quality and Reliability Engineering International, 29(3), pp. 345-356 (2013).

9. Haq, A. "A new hybrid exponentially weighted moving average control chart for monitoring process mean", Quality and Reliability Engineering International, 29(7), pp. 1015-1025 (2013).

10. Haq, A., Brown, J., and Moltchanova, E. "Improved fast initial response features for exponentially weighted moving average and cumulative sum control charts", Quality and Reliability Engineering International, 30(5), pp. 697-710 (2014).

11. McIntyre, G.A. "A method for unbiased selective sampling, using ranked sets", Crop and Pasture Science, 3(4), pp. 385-390 (1952).

12. Dell, T.R. and Clutter, J.L. "Ranked set sampling theory with order statistics background", The International Biometric Society, 28(2), pp. 545-555 (1972).

13. Stokes, S.L. "Ranked set sampling with concomitant variables", Communications in Statistics Theory and Methods, 6(12), pp. 1207-1211 (1977).

14. Samawi, H.M., Ahmed, M.S., and Abu-Dayyeh, W. "Estimating the population mean using extreme ranked set sampling", Biometrical Journal, 38(5), pp. 577-586 (1996).

15. Muttlak, H.A. "Median ranked set sampling", Journal of Applied Statistical Science, 6(4), pp. 245-255 (1997).

16. Muttlak, H.A. "Investigating the use of quartile ranked set samples for estimating the population mean", Applied Mathematics and Computation, 146(2-3), pp. 437-443 (2003).

17. Al-Nasser, A.D. "L ranked set sampling: A generalization procedure for robust visual sampling", Communications in Statistics - Simulation and Computation, 36(1), pp. 33-43 (2007).

18. Haq, A., Brown, J., Moltchanova, E., and Al-Omari, A.I. "Varied L ranked set sampling scheme", Journal of Statistical Theory and Practice, 9(4), pp. 741-767 (2015).

19. Salazar, R.D. and Sinha, A.K. "Control chart $\bar{x}$ based on ranked set sampling", Comunicacion Tecnica, No. 1-97-09 (PE/CIMAT) (1997).

20. Muttlak, H.A. and Al-Sabah, W. "Statistical quality control based on ranked set sampling", Journal of Applied Statistics, 30(9), pp. 1055-1078 (2003).

21. Abujiya, M.R. and Muttlak, H. "Quality control chart for the mean using double ranked set sampling", Journal of Applied Statistics, 31(10), pp. 1185-1201 (2004).

22. Al-Omari, A.I. and Haq, A. "Improved quality control charts for monitoring the process mean, using doubleranked set sampling methods", Journal of Applied Statistics, 39(4), pp. 745-763 (2012).

23. Al-Sabah, W.S. "Cumulative sum statistical control charts using ranked set sampling data", Pakistan Journal of Statistics, 26(2), pp. 365-378 (2010).
24. Abujiya, M.R., Riaz, M., and Lee, M.H. "Enhancing the performance of combined Shewhart-EWMA charts", Quality and Reliability Engineering International, 29(8), pp. 1093-1106 (2013).

25. Abujiya, M.R., Riaz, M., and Lee, M.H. "Improving the performance of combined Shewhart-cumulative sum control charts", Quality and Reliability Engineering International, 29(8), pp. 1193-1206 (2013).

26. Awais, M. and Haq, A. "An EWMA chart for monitoring process mean", Journal of Statistical Computation and Simulation, 88(5), pp. 1003-1025 (2018).

27. Awais, M. and Haq, A. "A new cumulative sum control chart for monitoring the process mean using varied L ranked set sampling", Journal of Industrial and Production Engineering, 35(2), pp. 74-90 (2018).

28. Haq, A. "An improved mean deviation exponentially weighted moving average control chart to monitor process dispersion under ranked set sampling", Journal of Statistical Computation and Simulation, 84(9), pp. 2011-2024 (2014).

29. Mehmood, R., Riaz, M., and Does, R.J.J.M. "Control charts for location based on different sampling schemes", Journal of Applied Statistics, 40(3), pp. 483494 (2013).

30. Mehmood, R., Riaz, M., and Does, R.J.J.M. "Quality quandaries: On the application of different ranked set sampling schemes", Quality Engineering, 26(3), pp. 370-378 (2014).

31. Haq, A., Brown, J., and Moltchanova, E. "New exponentially weighted moving average control charts for monitoring process dispersion", Quality and Reliability Engineering International, 30(8), pp. 1311-1332 (2014).

32. Haq, A., Brown, J., and Moltchanova, E. "A new maximum exponentially weighted moving average control chart for monitoring process mean and dispersion", Quality and Reliability Engineering International, 31(8), pp. 1587-1610 (2015).

33. Haq, A., Brown, J., and Moltchanova, E. "A new exponentially weighted moving average control chart for monitoring the process mean", Quality and Reliability Engineering International, 31(8), pp. 1623-1640 (2015).

34. Haq, A., Brown, J., and Moltchanova, E. "A new maximum exponentially weighted moving average control chart for monitoring process mean and dispersion", Quality and Reliability Engineering International, 31(8), pp. 1587-1610 (2015).

35. Haq, A., Brown, J., and Moltchanova, E. "New synthetic control charts for monitoring process mean and process dispersion", Quality and Reliability Engineering International, 31(8), pp. 1305-1325 (2015).

36. Haq, A., Brown, J., Moltchanova, E., and Al-Omari, A.I. "Effect of measurement error on exponentially weighted moving average control charts under ranked set sampling schemes", Journal of Statistical Computation and Simulation, 85(6), pp. 1224-1246 (2015). 
37. Abbasi, S.A. and Riaz, M. "On dual use of auxiliary information for efficient monitoring", Quality and Reliability Engineering International, 32(2), pp. 705-714 (2016).

38. Abid, M., Nazir, H.Z., Riaz, M., and Lin, Z. "Use of ranked set sampling in nonparametric control charts", Journal of the Chinese Institute of Engineers, 39(5), pp. 627-636 (2016).

39. Abid, M., Nazir, H.Z., Riaz, M., and Lin, Z. "Investigating the impact of ranked set sampling in nonparametric CUSUM control charts", Quality and Reliability Engineering International, 33(1), pp. 203214 (2016).

40. Munir, W. and Haq, A. "New cumulative sum control charts for monitoring process variability", Journal of Statistical Computation and Simulation, 87(15), pp. 2882-2899 (2017).

41. Rhoads, T.R., Montgomery, D.C., and Mastrangelo, C.M. "A fast initial response scheme for the exponentially weighted moving average control chart", Quality Engineering, 9(2), pp. 317-327 (1996).

42. Steiner, S.H. "EWMA control charts with time-varying control limits and fast initial response", Journal of Quality Technology, 31(1), pp. 75-86 (1999).

43. David, H.A. and Nagaraja, H.N., Order Statistics, 3rd Edn., John Wiley \& Sons, Inc., Hoboken, New Jersey (2003).

44. Montgomery, D.C., Introduction to Statistical Quality Control, 6th Edn., John Wiley \& Sons, Inc., New York (2007).

\section{Biographies}

Muhammad Awais obtained his MSc and MPhil degrees in Statistics from the Department of Statistics, Quaid-i-Azam University, Islamabad, Pakistan. His research interest is in statistical process control.

Abdul Haq graduated (PhD) from the School of Mathematics and Statistics, University of Canterbury, Christchurch, New Zealand. He is an Assistant Professor at the Department of Statistics, Quaid-i-Azam University, Islamabad, Pakistan. His research interest is in statistical process control. 